\title{
NEAR-IR IMAGING POLARIMETRY TOWARD A BRIGHT-RIMMED CLOUD: MAGNETIC FIELD IN SFO 74
}

\author{
Takayoshi Kusune ${ }^{1}$, Koji Sugitani ${ }^{1}$, JingQi Miao ${ }^{2}$, Motohide Tamura ${ }^{3,4}$, Yaeko Sato ${ }^{4}$, Jungmi Kwon ${ }^{3,4}$, \\ Makoto Watanabe ${ }^{5}$, Shogo Nishiyama ${ }^{6}$, Takahiro Nagayama ${ }^{7}$, and Shuji Sato ${ }^{8}$ \\ ${ }^{1}$ Graduate School of Natural Sciences, Nagoya City University, Mizuho-ku, Nagoya 467-8501, Japan \\ ${ }^{2}$ Centre for Astrophysics and Planetary Science, School of Physical Sciences, University of Kent, Canterbury, Kent CT2 7NR, UK \\ ${ }^{3}$ Department of Astronomy, University of Tokyo, 7-3-1 Hongo, Bunkyo-ku, Tokyo 113-0033, Japan \\ ${ }^{4}$ National Astronomical Observatory, 2-21-1 Osawa, Mikata, Tokyo 181-8588, Japan \\ ${ }^{5}$ Department of Cosmosciences, Hokkaido University, Kita 10, Nishi 8, Kita-ku, Sapporo, Hokkaido 060-0810, Japan \\ ${ }^{6}$ Faculty of Education, Miyagi University of Education, Sendai 980-0845, Japan \\ ${ }^{7}$ Department of Physics, Kagoshima University, 1-21-35 Korimoto, Kagoshima 890-0065, Japan \\ ${ }^{8}$ Department of Astrophysics, Nagoya University, Chikusa-ku, Nagoya 464-8602, Japan \\ Received 2013 May 4; accepted 2014 October 26; published 2014 December 19
}

\begin{abstract}
We have made near-infrared $\left(J H K_{\mathrm{s}}\right)$ imaging polarimetry of a bright-rimmed cloud (SFO 74$)$. The polarization vector maps clearly show that the magnetic field in the layer just behind the bright rim is running along the rim, quite different from its ambient magnetic field. The direction of the magnetic field just behind the tip rim is almost perpendicular to that of the incident UV radiation, and the magnetic field configuration appears to be symmetric as a whole with respect to the cloud symmetry axis. We estimated the column and number densities in the two regions (just inside and far inside the tip rim) and then derived the magnetic field strength, applying the Chandrasekhar-Fermi method. The estimated magnetic field strength just inside the tip rim, $\sim 90 \mu \mathrm{G}$, is stronger than that far inside, $\sim 30 \mu \mathrm{G}$. This suggests that the magnetic field strength just inside the tip rim is enhanced by the UV-radiation-induced shock. The shock increases the density within the top layer around the tip and thus increases the strength of the magnetic field. The magnetic pressure seems to be comparable to the turbulent one just inside the tip rim, implying a significant contribution of the magnetic field to the total internal pressure. The mass-to-flux ratio was estimated to be close to the critical value just inside the tip rim. We speculate that the flat-topped bright rim of SFO 74 could be formed by the magnetic field effect.
\end{abstract}

Key words: infrared: stars - ISM: individual objects (SFO 74) - ISM: magnetic fields ISM: structure - polarization

\section{INTRODUCTION}

Bright-rimmed clouds (BRCs), which are located at the periphery of $\mathrm{H}$ II regions, are considered to be potential sites for induced star formation by UV radiation from nearby OB stars (e.g., Elmegreen 1998, 2011 and references therein). To understand dynamical evolution of such molecular clouds, many theorists have developed 2D/3D hydrodynamical models. They reproduced successfully some of the observed properties of BRCs. However, most simulations did not always include all of the related physical effects, particularly the magnetic field effect (e.g., Lefloch \& Lazareff 1994; White et al. 1999; Williams et al. 2001; Kessel-Deynet \& Burkert 2003; Miao et al. 2009, 2010; Mackey $\&$ Lim 2010). Miao et al. (2006) did include the magnetic field effect in their 3D smoothed particle hydrodynamic simulation by adding a magnetic field pressure in the equation of state. However, evolution of the magnetic field direction cannot be inferred from their simulation. In fact, comparing the ambipolar diffusion timescale $(\sim 10 \mathrm{Myr})$ with the radiation-driven implosion (RDI) shock formation ( $\sim 0.05-0.08 \mathrm{Myr})$ and a BRC formation time (0.1-0.5 Myr), it is not difficult to see that the magnetic field effect may not be able to produce a generally significant influence on the dynamics of the BRC because of the extremely low concentration of the ions/electrons in the main body of the BRC. The magnetic field may play a certain role in the morphological formation of a BRC. Recently, Mackey \& Lim (2011) performed 3D magnetohydrodynamic simulations and suggested that only a strong magnetic field could significantly alter the nonmagnetized dynamics. More recently, Motoyama et al. (2013) modeled a photoevaporating cloud, including the magnetic field effect, and suggested that the magnetic pressure might play an important role in the cloud density structure. It is obvious that the physical effects, particularly the magnetic field, are not treated properly in simulations. Thus, it is of crucial importance to obtain information on magnetic fields in and around BRCs.

Wide-field near-infrared (NIR) polarimetry is a good tool for measuring the magnetic field direction toward molecular clouds (e.g., Tamura et al. 2007), and Sugitani et al. (2007) made NIR imaging polarimetry of molecular pillars with bright rims in M16. Their polarization observations show that magnetic fields are aligned along some of the pillars in M16, probably as a result of the UV impact from its exciting stars, while the global magnetic structure around M16 is quite different from this alignment. However, such measurements for the magnetic field toward BRCs are still rare.

Eighty-nine BRCs associated with IRAS point sources were cataloged by Sugitani et al. (1991) and Sugitani \& Ogura (1994) in the northern and southern hemispheres and are referred to as the SFO objects in SIMBAD. The levels of star formation of 45 BRCs in the southern hemisphere were examined by Urquhart et al. (2009) with CO and mid-infrared data, as well as the radio continuum data of Thompson et al. (2004). They identified 24 of them that show strong interaction with H II regions. We started a systematic study of these BRCs and have so far examined the magnetic fields toward eight BRCs by making a single field observation $(\sim 7$ '.7 $\times 7$ '.7) of NIR polarimetry for each BRC. In the course of this study, we found that the magnetic field of SFO 74 was most clearly revealed among these eight BRCs. Thus, in this paper we report 

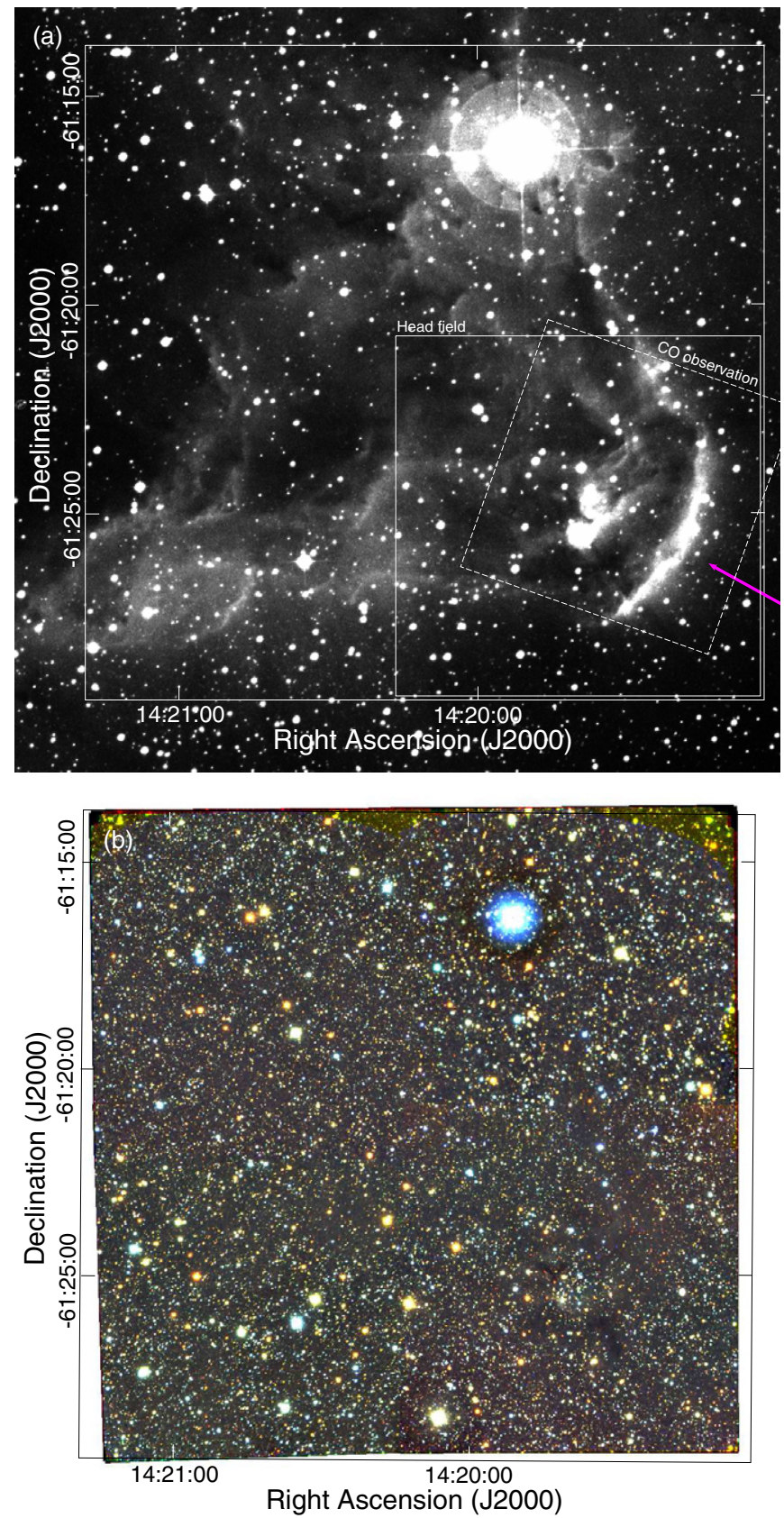

Figure 1. (a) SuperCOSMOS $\mathrm{H}_{\alpha}$ image of SFO 74. The incident UV radiation direction from HD 124314, which is identified as the main exciting star of RCW 85/SFO 74 (O6 V; Yamaguchi et al. 1999b); $\sim 60^{\circ}$ in P.A. is shown with a purple arrow. The area of our NIR imaging polarimetry (see Figure 1(b)) is shown by a filled square with coordinates. The head field (each square in Figures 6 and 8-10) is also shown by a small, filled square. The area of CO observation is also shown by a dashed square. (b) Three-color composite, mosaic $(2 \times 2)$ image of SFO 74 $\left(J\right.$, blue; $H$, green; $K_{\mathrm{s}}$, red). The size of the area is $\sim 16^{\prime} .0 \times 16^{\prime} .0$. The yellow color regions at the upper edge are due to the dead pixels of the $J$-band array.

the results by adding three more fields around its head field (see Figure 1).

SFO 74 is situated in the H II region RCW 85 ( $d \sim 1.5 \mathrm{kpc}$; Georgelin et al. 1987). HD 124314 is identified as the main exciting star of RCW 85/SFO 74 (O6 V; Yamaguchi et al. $1999 \mathrm{~b}$ ), with location at the projected distance of $\sim 15 \mathrm{pc}$ from SFO 74. We estimated a relatively weak UV photon flux of $\sim 4.5 \times 10^{8} \mathrm{~cm}^{-2} \mathrm{~s}^{-1}$, assuming a distance of $\sim 15 \mathrm{pc}$ from the O6 V star (Panagia 1973), nearly the same as that by Urquhart et al. (2009). The point source of IRAS 141596111 (MSX G313.2850-00.3350) is located in the head of SFO 74, where Thompson et al. (2004) reported the presence of an ultracompact (UC) H II region. This BRC was classified morphologically as Type A (moderately curved rim; Sugitani \& Ogura 1994) by focusing on just its brighter tip part. As seen in the SuperCOSMOS $\mathrm{H}_{\alpha}$ image (Parker et al. 2005) of Figure 1(a), however, its entire shape seems to be Type B (tightly curved rim) rather than Type A. This shape appears to be symmetric with respect to the UV incident direction from the exciting stars, so it is very likely that this shape was formed by UV radiation, i.e., the RDI effect by massive stars (e.g., Miao et al. 2009).

In this paper we concentrate on the results of our NIR polarization measurements of SFO 74, including those of the $\mathrm{CO}$ archival data. In Section 2 we describe our NIR observations and $\mathrm{CO}$ archival data. In Section 3 we present the results of the polarimetry and CO data. In Section 4 we discuss (1) the cloud structure and the magnetic field configuration, (2) the physical quantities related to the magnetic field, (3) the flat-topped shape of SFO 74, and (4) star formation activity. A summary is given in Section 5. Our polarimetric study of other BRCs will be presented in the near future.

\section{OBSERVATIONS AND ARCHIVAL DATA}

\subsection{Near-infrared Observations}

$J H K_{\text {s }}$ polarimetric observations of SFO 74 were made on 2012 February 25 for the head field (a small filled square in Figure 1(a)). Three more fields (north, northeast, and east of the head field) were added on 2013 March 6, 10, and 12 and 2014 April 20. The mosaic image obtained is shown in Figure 1(b). The consistency of the polarization angles and degrees was checked among data taken for these two years of runs in 2013 and 2014 (see Appendix A).

We used the imaging polarimeter SIRPOL (polarimetry mode of the SIRIUS camera: Kandori et al. 2006) mounted on the Infrared Survey Facility (IRSF) $1.4 \mathrm{~m}$ telescope at the South African Astronomical Observatory. The SIRIUS camera is equipped with three $1024 \times 1024 \mathrm{HgCdTe}$ (HAWAII) arrays, $J H K_{\mathrm{s}}$ filters, and dichroic mirrors, which enable simultaneous $J H K_{\mathrm{S}}$ observations (Nagashima et al. 1999; Nagayama et al. 2003 ). The field of view at each band is $\sim 7.7 \times 7.7$ with a pixel scale of 0.45 .

We obtained 10 dithered exposures, each $15 \mathrm{~s}$ long, at four wave-plate angles $\left(0^{\circ}, 22.5,45^{\circ}\right.$, and 67.5 in the instrumental coordinate system) as one set of observations and repeated this six times. Eventually, the total on-target exposure times were 900 s per each wave-plate angle. Self-sky images were used for median sky subtraction. The average seeing size ranged from $\sim 1^{\prime \prime} .2$ to $1^{\prime \prime} .7$ at $K_{\mathrm{s}}$ during the observations. Twilight flatfield images were obtained at the beginning and/or end of the observations.

\subsection{CO Archival Data}

The ${ }^{12} \mathrm{CO},{ }^{13} \mathrm{CO}$, and $\mathrm{C}^{18} \mathrm{O}(1-0)$ data of the Mopra telescope were taken from the Australia Telescope Online Archive (Project Codes: M126; Source Name: SFO 74) in order to reveal the structure of the cloud and to evaluate the velocity dispersions of molecular gas. The data are available only within a square area of $\sim 6^{\prime} .0 \times 6^{\prime} .0$ toward the cloud head (the dashed square in Figure 1(a)) and were reduced using Gridzilla/Livedata. ${ }^{9}$

\footnotetext{
9 http://www.atnf.csiro.au/computing/software/livedata/
} 
According to the website (Information for Mopra observers ${ }^{10}$ ), the beamwidth (FWMH) is $\sim 30^{\prime \prime}$, the antenna efficiency is $\sim 0.4$, and the velocity resolution is $\sim 0.088 \mathrm{~km} \mathrm{~s}^{-1}$ at $115 \mathrm{GHz}$.

\section{RESULTS}

\subsection{Photometry and Source Selection}

Standard reduction procedures were applied with IRAF. Aperture polarimetry was performed at $J, H$, and $K_{\mathrm{s}}$ with an aperture radius of about FWHM size by using APHOT of the DAOPHOT package. The Two Micron All Sky Survey (2MASS) catalog (Skrutskie et al. 2006) was used for absolute photometric calibration. We calculated the Stokes parameters as follows: $Q=I_{0}-I_{45}, U=I_{22.5}-I_{67.5}$, and $I=$ $\left(I_{0}+I_{22.5}+I_{45}+I_{67.5}\right) / 2$, where $I_{0}, I_{22.5}, I_{45}$, and $I_{67.5}$ are intensities at four wave-plate angles. To obtain the Stokes parameters in the equatorial coordinate system $\left(Q^{\prime}\right.$ and $\left.U^{\prime}\right)$, a rotation of $105^{\circ}$ (Kandori et al. 2006, and see also Appendix B) was applied to them. We calculated the degree of polarization $P$ and the polarization angle $\theta$ as follows: $P=\sqrt{Q^{2}+U^{2}} / I$ and $\theta=(1 / 2) \tan ^{-1}\left(U^{\prime} / Q^{\prime}\right)$. The absolute accuracy of the position angle (P.A.) of polarization was estimated to be better than $3^{\circ}$ (Kandori et al. 2006), where P.A. is the angle measured from north in an easterly direction. The polarization efficiencies were estimated to be $95.5 \%, 96.3 \%$, and $98.5 \%$ at $J, H$, and $K_{\mathrm{s}}$, respectively, and the measurable polarization is $\sim 0.3 \%$ all over the field of view at each band (Kandori et al. 2006). With these high polarization efficiencies and low instrument polarization, no particular corrections were made here. The polarization measurement error $(\Delta P)$ and the P.A. error $(\Delta \theta)$ were calculated from the photometric errors, and the degrees of polarization were debiased as $P_{\text {debias }}=\sqrt{P^{2}-\Delta P^{2}}$ (Wardle \& Kronberg 1974). Hereafter, we use $P$ as a substitute for this debiased value. In consideration of the above measurable polarization of $\sim 0.3 \%$, for the sources with the polarization measurement errors of $<0.3 \%$ we adopt $0.3 \%$ as their polarization errors when we calculate $\Delta \theta$. We confirmed an agreement of the polarization angle within their errors between two independent observations for almost all the stars that were located commonly in the neighboring two fields.

We have measured $J, H$, and $K_{\mathrm{s}}$ polarization for point sources to examine the magnetic field structure. Only the sources with photometric errors of $<0.1 \mathrm{mag}$ were used for analysis. We excluded sources plotted redward of the reddening line from the A0 star on the $J-H$ versus $H-K_{\text {s }}$ diagram (Figure 2) and/or those associated with reflection nebulae as young stellar object candidates. As seen in this color-color diagram, most of the detected sources seem to be giants or reddened giants. Here we adopted the reddening line of $E(\mathrm{~J}-\mathrm{H}) / E(\mathrm{H}-\mathrm{K}) \sim 1.95$ (Chini \& Wargau 1998), because this value seems to fit to the distribution of the reddened sources in Figure 2.

We regard the polarization of these point sources as the polarization of the dichroic origin and assume the polarization vector to represent the direction of the local magnetic field averaged over the line of sight to the source. We made polarization degree $P$ versus $H-K_{\mathrm{s}}$ color diagrams for point sources with polarization measurement errors of $<0.3 \%$ (Figure 3 ). For the $J$-band panel we include only the sources detected at all three bands, but for the $H$ - and $K_{\mathrm{s}}$-band panels we include the sources detected at both the $H$ and $K_{\mathrm{s}}$ bands. We obtained the best-fit linear lines $a_{J}=P(J) /\left[\left(H-K_{\mathrm{s}}\right)-0.15\right], a_{H}=P(H) /\left[\left(H-K_{\mathrm{s}}\right)-0.15\right]$,

\footnotetext{
10 http://www.narrabri.atnf.csiro.au/mopra/obsinfo.html
}

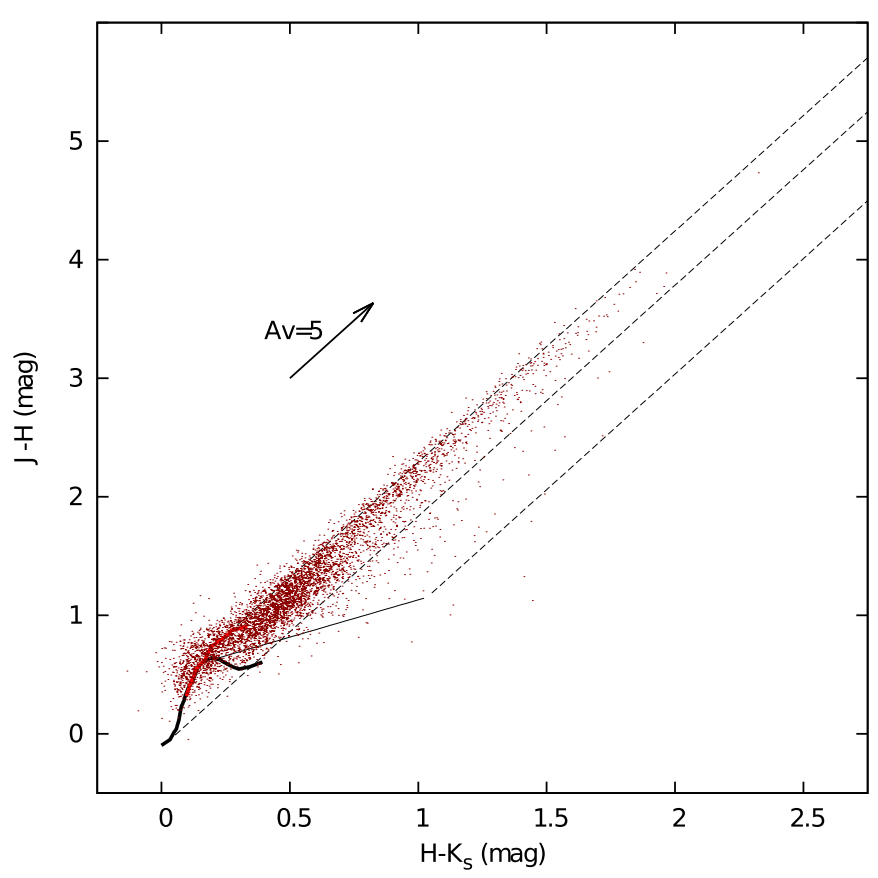

Figure 2. $J-H$ vs. $H-K_{\mathrm{s}}$ color-color diagram toward SFO 74. The thick curves are the loci of dwarfs (black line) and giants (red line). The data for A0-M6 dwarfs and G0-M7 giants are from Bessell \& Brett (1988). The unreddened CTTS locus of Meyer et al. (1997) is also denoted by the thin solid line. These data are transformed to the 2MASS system by using the equations in the explanatory supplement (http://www.ipac.caltech.edu/2mass/releases/ allsky/doc/sec6_4b.html by R. M. Cutri et al.). The three dashed lines are reddening ones parallel to the reddening vector $\left(E(J-H) / E\left(H-K_{\mathrm{S}}\right)=1.95\right.$; Chini \& Wargau 1998).

and $a_{K_{\mathrm{s}}}=P\left(K_{\mathrm{s}}\right) /\left[\left(H-K_{\mathrm{s}}\right)-0.15\right]$ (see Figure 3 ) assuming the intrinsic color $(H-K \mathrm{~s})_{0}=0.15$ of background sources based on the model calculations by Wainscoat et al. (1992).

As Kusakabe et al. (2008) mentioned, all of the observed NIR polarizations are not originated by interstellar polarization, i.e., there are highly polarized sources that cannot be explained by interstellar origin, but by their circumstellar structures such as disks and envelopes. In order to exclude the point sources with large polarization degrees that are not considered to be of dichroic origin, we set an upper limit for the interstellar polarization. On the other hand, if the source has intrinsic polarization or background polarization that had occurred in the more distant area, and if its polarization direction is different from that of the local magnetic field of interest, its polarization degree might become small. We also set a lower limit to exclude such sources. In Figure 3, the slopes of the dashed lines of each panel are double and one-half of the slope of the best-fit linear line, and most of the plotted sources lie between these two dashed lines. Therefore, we adopted them as the upper and lower limits of the polarization efficiency. Only the sources between the two limits and of $P / \Delta P>3.0$, corresponding to $\Delta \theta \lesssim 10^{\circ}$, were used for plotting polarization vectors.

We can see a clump of stars located blueward of each adopted upper limit line in Figure 3, e.g., the one located at $E\left(H-K_{\mathrm{s}}\right) \lesssim 0.2$ on the $H$-band panel. Most stars of the clump are visible in the optical image, so they are likely to be foreground dwarfs and contain no information on the magnetic field of SFO 74. Thus, the clump indicates small foreground polarization at $H$, so we safely excluded these clumps from the sample. 

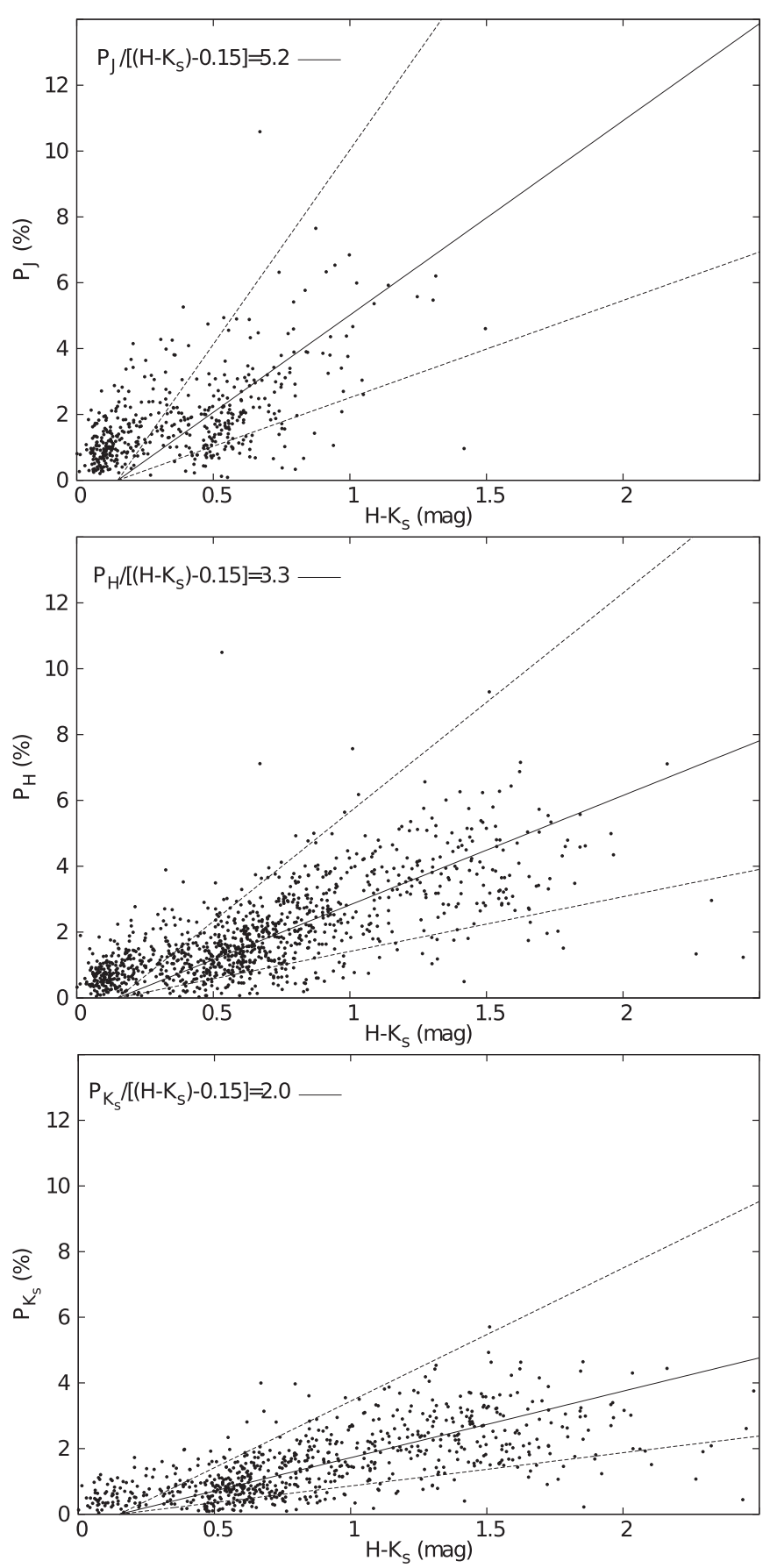

Figure 3. Polarization degree vs. $H-K_{\mathrm{s}}$ color diagrams at $J, H$, and $K_{\mathrm{s}}$ for sources having a polarization measurement error of $<0.3 \%$. The best-fit linear lines obtained for the sources with $H-K_{\mathrm{s}}>0.15$ are shown by solid lines in each panel. The dashed lines are double and half of the best-fit lines and are the lines of our adopted maximum or minimum polarization efficiency.

\subsection{Polarization Data}

Figure 4 shows a $J$-band polarization vector map superposed on the SuperCOSMOS H $\alpha$ image of SFO 74. SFO 74 is clearly visible in optical, so polarization, as well as extinction, is mostly caused by the cloud or background, not foreground interstellar medium.

In Figure 4, the magnetic field directions inside the cloud seem different from those outside of the cloud (toward the northern, western, and southern edges). We constructed the histograms of P.A. for $J$-, $H$-, and $K_{\mathrm{s}}$-band polarization vectors over the entire observed area (Figure 5). These histograms have a peak of $\sim 95^{\circ}$ in P.A., indicating the direction of the ambient magnetic field. The longer the observed wavelength is, the clearer this peak becomes. This is because the extinction, i.e., the polarization degree, decreases at longer wavelengths. Then, most of the polarization angles at $H$ and $K_{\mathrm{s}}$ are likely indicating the direction of the background magnetic field.

Inside the cloud, the polarization vectors follow two patterns: (1) following the curved bright rim just behind the bright rim, and (2) parallel to an approximate symmetry axis of the cloud in the area away from the bright-rim tip. As the symmetry axis, we adopt an extension of the line (purple solid line in Figure 4) connecting the position of HD 124314 and the middle point of the bright-rim tip of the cloud (dashed line in Figure 4). These patterns of the magnetic field configuration are even clearer in Figure 6, showing $H$ - and $K_{\mathrm{s}}$-band polarization vector maps toward the head region of SFO 74.

Figure 7 shows the change of P.A.s of $H$-band polarization along the symmetric axis. We used the sources within the white dashed box in Figure 7(a), and the horizontal axis of Figure 7(b) represents a position $(x)$ from the bright-rim tip, where the position direction is the tail side of the cloud. In a range of $x \sim-2^{\prime}$ to $0^{\prime}$, an average P.A. is $\sim 93^{\circ}$, which is identical to the direction of the ambient magnetic field. The P.A.s in the tip region just behind the bright rim $\left(x \sim 0^{\prime}-2^{\prime}\right)$ seem to scatter around $140^{\circ}$, and their average is $\sim 141^{\circ}$, nearly perpendicular to the incident direction of UV radiation, $\sim 60^{\circ}$. In a range of $x \sim 2^{\prime}-4^{\prime}$, P.A.s decline drastically from $\sim 141^{\circ}$ to $\sim 70^{\circ}$. In $x \sim$ $4^{\prime}-6^{\prime}$, an average P.A. is $\sim 68^{\circ}$, nearly parallel to the symmetric axis with some scatter. In $x \sim 6^{\prime}-9^{\prime}$, P.A.s gradually increase from $\sim 70^{\circ}$ to $\sim 90^{\circ}$ and remain constant around $\sim 90^{\circ}$ thereafter. In $x \gtrsim 9^{\prime}$, most P.A.s seem to be around $\sim 90^{\circ}$, close to that of the ambient magnetic field, although there are a certain number of sources with P.A. $\sim 60^{\circ}$. Excluding the sources with P.A. $<70^{\circ}$, an average P.A. is calculated to be $\sim 86^{\circ}$ in $x \sim 9^{\prime}-14^{\prime}$, differing only by $\sim 9^{\circ}$ relative to that of the ambient magnetic field. Thus, this region with $x \gtrsim 9^{\prime}$ seems to be dominated by the background polarization component, while the region with $x \sim 0^{\prime}-6^{\prime}$ seems to be dominated by the cloud polarization one. The intermediate region with $x \sim 6^{\prime}-9^{\prime}$ can be considered the transition region.

\subsection{CO Data}

The maps of the ${ }^{12} \mathrm{CO}$ peak antenna temperature $\left(T_{\mathrm{A}}^{*}\right)$, the ${ }^{13} \mathrm{CO}$ integrated intensity, and the ${ }^{13} \mathrm{CO}$ velocity width are shown in Figure 8. ${ }^{11}$ The peak $T_{\mathrm{A}}^{*}$ was determined by Gaussian fitting of ${ }^{12} \mathrm{CO}$ spectra for the points detected above the $5 \sigma$ level, and the maximum value is $\sim 28 \mathrm{~K}$ on the peak $T_{\mathrm{A}}^{*}$ map. We derived the excitation temperature $T_{\mathrm{ex}}$ and the ${ }^{13} \mathrm{CO}$ column density $N_{13} \mathrm{CO}$, following the equations of Yamaguchi et al. (1999a) with the antenna efficiency of 0.4. We calculated the column density $N_{\mathrm{H}_{2}}$, adopting the column density ratio of $\mathrm{H}_{2}$ to ${ }^{13} \mathrm{CO}$ as $\sim 5.0 \times 10^{5}$ (Dickman 1978). The peak column density derived is $\sim 5.1 \times 10^{22} \mathrm{~cm}^{-2}$ at the peak of the ${ }^{13} \mathrm{CO}$ integrated intensity, and the column density distribution is very similar to the integrated intensity distribution in morphology. Hence, we do not show the column density map here. The total mass toward the observed was estimated to be $\sim 1400 M_{\odot}$, which is a bit larger than that of $\sim 1000 M_{\odot}$ estimated by Yamaguchi

\footnotetext{
11 Toward this area, two strong velocity components of $\sim-30 \mathrm{~km} \mathrm{~s}^{-1}$ and $\sim-50 \mathrm{~km} \mathrm{~s}^{-1}$ and other minor components are detectable. Here we focus on only the $\sim-30 \mathrm{~km} \mathrm{~s}^{-1}$ component of SFO 74 .
} 


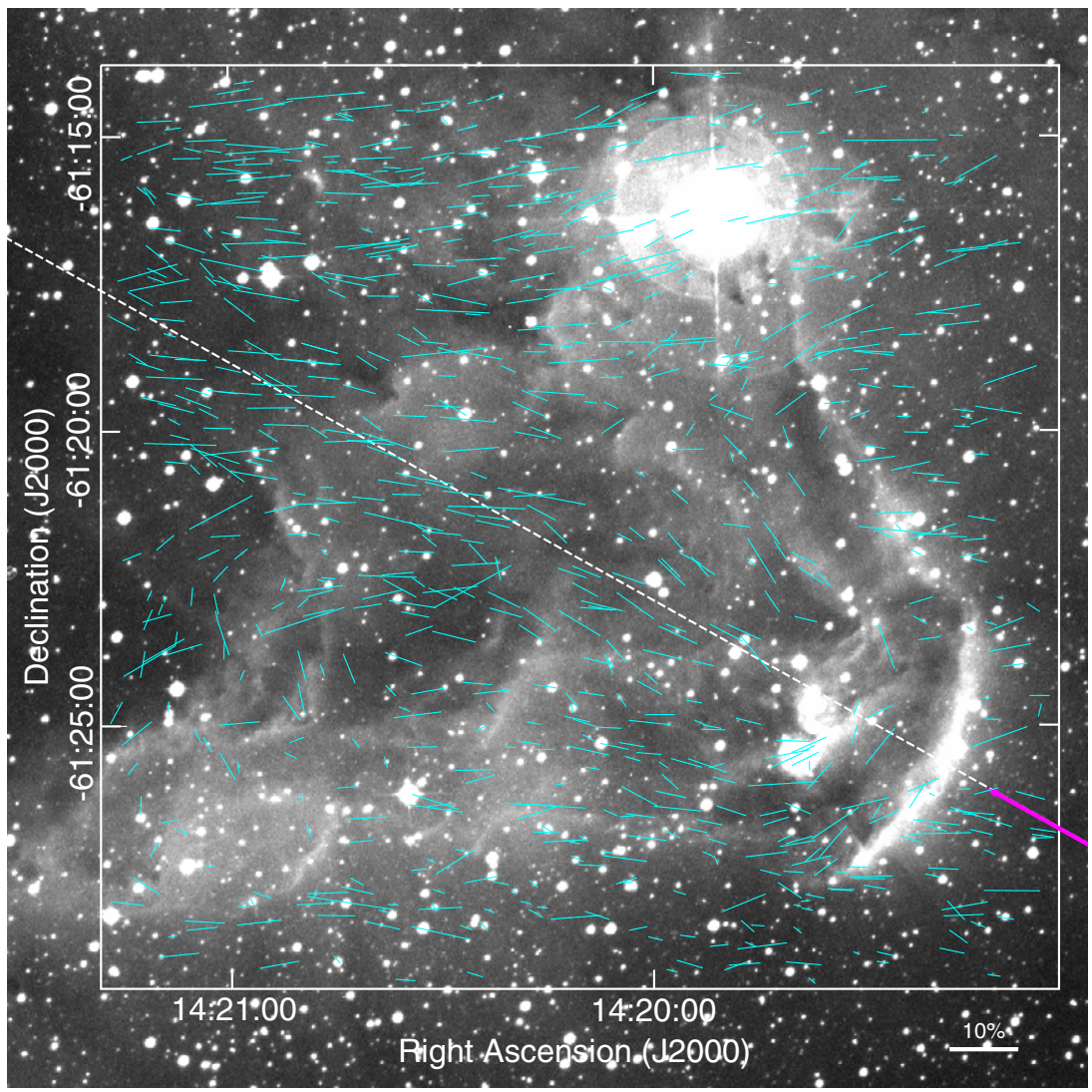

Figure 4. $J$-band polarization vector map of SFO 74 superposed on the SuperCOSMOS $\mathrm{H}_{\alpha}$ image. A $10 \%$ vector is shown at the bottom right. The incident UV radiation direction is shown with a purple arrow, and its extended line (our adopted approximately symmetry axis) is shown by a white dashed line.

et al. (1999b). The ${ }^{13} \mathrm{CO}$ velocity width was determined by Gaussian fitting of ${ }^{13} \mathrm{CO}$ spectra for the points detected above the $5 \sigma$ level.

In $\mathrm{CO}$, the SFO 74 cloud is seen inside the bright rim, and its edge on the exciting star side agrees well with the bright-rim tip. In the $T_{\mathrm{A}}^{*}$ map, the temperature is the highest just behind the bright-rim tip and gradually decreases with distance away from the bright-rim tip. The derived maximum $T_{\text {ex }}$ is $\sim 70 \mathrm{~K}$, which suggests that the cloud temperature is much higher than that in typical dark clouds. The peak positions are slightly different between two maps (Figures 8(a) and (b)) with a separation of $\sim 1^{\prime}$, and the peak in the $T_{\mathrm{A}}^{*}$ map is closer to the bright-rim tip. The IRAS source is not located toward the peak of the $T_{\mathrm{A}}^{*}$ map or that of the ${ }^{13} \mathrm{CO}$ integrated intensity map. In Figure 8(c), the velocity width is small in the area that is elongated along/ just behind the bright-rim tip, with an almost uniform value of $\sim 1.4 \mathrm{~km} \mathrm{~s}^{-1}$. The velocity width increases with distance away from the bright-rim tip but eventually decreases. The areas of the largest velocity width are located $\sim 2^{\prime}$ from the bright-rim tip and do not coincide with the two peaks of the ${ }^{12} \mathrm{CO} T_{\mathrm{A}}^{*}$ and ${ }^{13} \mathrm{CO}$ integrated intensity maps.

Figure 9 shows the channel maps of ${ }^{13} \mathrm{CO}$. The LSR central velocity ranges from $-31.17 \mathrm{~km} \mathrm{~s}^{-1}$ up to $-26.13 \mathrm{~km} \mathrm{~s}^{-1}$, with a velocity interval of $0.46 \mathrm{~km} \mathrm{~s}^{-1}$. With an increase in velocity, the mushroom-like structure (a core + earlike structure at the two sides of the core) becomes visible, most clearly at $-28.88 \mathrm{~km} \mathrm{~s}^{-1}$. At the channels over $-27.96 \mathrm{~km} \mathrm{~s}^{-1}$, another component appears $\sim 2^{\prime}$ east-southeast of the peak position seen at $-28.88 \mathrm{~km} \mathrm{~s}^{-1}$. In Figure 10(a), we present the two velocity components superposed on the Spitzer image by showing two integrated intensity maps with different velocity ranges.
Hereafter we refer to the blue component of $\sim-29 \mathrm{~km} \mathrm{~s}^{-1}$ as Cloud $\mathrm{A}$ and to the red component of $\sim-27.5 \mathrm{~km} \mathrm{~s}^{-1}$ as Cloud B. The IRAS source is located toward Cloud B, and a bright mid-IR nebula of the Spitzer image is also seen toward Cloud B. We believe that Cloud B is located at the near side of Cloud A because a dark area that may correspond to Cloud B is seen in the optical image (Figure 10(b)).

\section{ANALYSIS AND DISCUSSION}

\subsection{Cloud A Structure and Magnetic Field Configuration}

The ${ }^{13} \mathrm{CO}$ channel maps (Figure 9) show the mushroom structure consisting of the core and the ears. The part of the ears has higher redshifted velocity components with respect to the main velocity component of SFO 74 (see the two panels of $-30.71 \mathrm{~km} \mathrm{~s}^{-1}$ and $-30.25 \mathrm{~km} \mathrm{~s}^{-1}$ ). This structure appears to be consistent with the illustration derived from the 3D simulation results of RDI (Figure 5 of Miao et al. 2009), indicating that the SFO 74 structure had been formed by RDI. As already mentioned above, the temperature appears to decrease with distance away from the bright-rim tip, and no sign of star formation is seen at the peak position of the $T_{\mathrm{A}}^{*}$ map (see the details in Section 4.4). These represent that Cloud A is externally heated by the exciting stars of RCW 85 and support the idea that the RDI process has been going on.

The magnetic field configuration must have been also affected by RDI. In the very early stage of RDI, the magnetic field lines of Cloud A might be the same as those of the ambient magnetic field. When the top layer gas is ionized by the UV radiation of the exciting stars of RCW 85, a strong shock is induced by the ionizing/heating and propagates into the cloud. Then the surface 

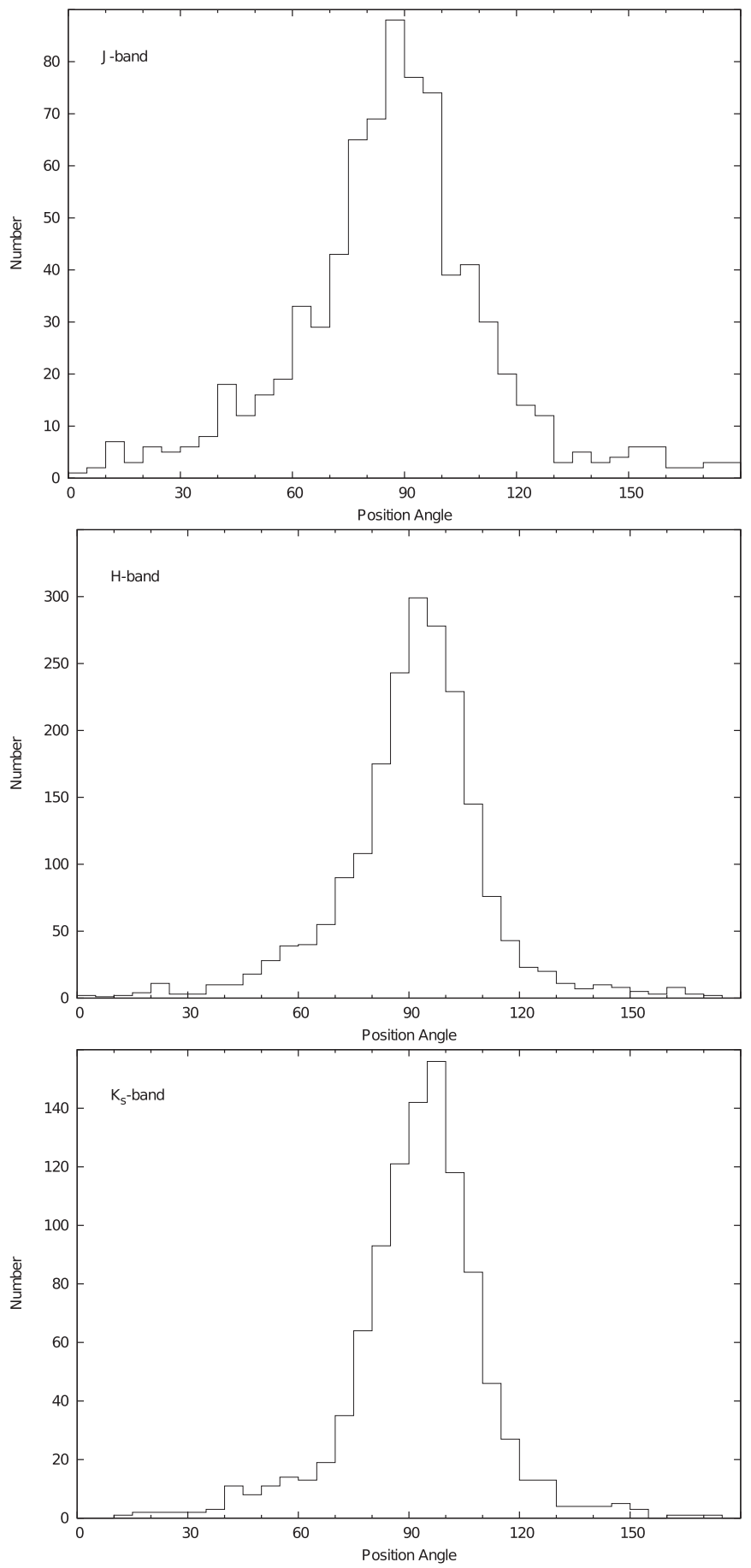

Figure 5. Histograms for the polarization P.A.s for point sources of $P / \Delta P>$ 3.0. Only the sources lie between our adapted maximum and minimum limits of the polarization efficiencies at $J, H$, and $K_{\mathrm{s}}$ band (see Section 3.1). For $J$ band, all the sources with polarization vectors in Figure 4 are used here.

layer of the cloud gets strongly compressed, which causes a magnetic field line to form an open loop, as shown in Figure 11. The magnetic field line of the loop looks almost perpendicular to the UV radiation direction. The looped magnetic field lines in the condensed layer form a stream of fragment lines around the bright rim, as seen from Figure 6. In Section 4.3 we prove that the magnetic pressure could further enhance this field line looping effect. With the further propagation into the cloud, although the shock becomes weaker, it continues driving the gas to converge toward the symmetric axis and eventually to form a dense core-tail structure of type B BRCs. Farther from the bright rim, the shock effect decreases its effect on gas compression (magnetic enhancement). We conclude that the magnetic field configuration of SFO 74 is a result of RDI.

\subsection{Physical Quantities}

We try to estimate the physical quantities related to the magnetic fields in the two regions; one is the tip region (Region 1) just behind the bright-rim tip, and the other is the inside region (Region 2) on the cloud symmetric axis. Regions 1 and 2 are shown by a red trapezoid and a green square, respectively, in Figure 7(a). Estimating the magnetic field strength with the CF (Chandrasekhar \& Fermi 1953) method requires an area where the magnetic field is uniform. Since the polarization vectors of Region 1 appear to uniformly follow the curve of the bright-rim tip (Figure 6), we selected Region 1, which is considered to be the compressed layer by RDI as discussed in Section 4.1. Since the magnetic field of Region 2 seems to be rather uniform, as shown in Section 3.2, we selected Region 2 so that its area is the same as that of Region 1, well outside Cloud B. The southwest half of Region 2 is covered by the $\mathrm{CO}$ observations, and we can crudely evaluate the velocity dispersion of Region 2. Here we use only the sources with $H$ and/or $K_{\mathrm{s}}$ vectors in Figure 6.

For Region 1, we adopt a thick, uniform disk approximation and assume that we view this disk edge-on. In this case, the density can be obtained by dividing the central/maximum column density toward the axis by the diameter/width of the disk, i.e., the depth. The average of color excess $E\left(H-K_{\mathrm{s}}\right)$ is $\sim 1.5 \pm 0.7 \mathrm{mag}$ for 20 sources detected toward the central part of Region 1 (within 60" of the symmetric axis), and $N_{\mathrm{H}_{2}} \sim 2.9 \times 10^{22} \mathrm{~cm}^{-2}$ is roughly obtained, using the reddening law, $E\left(H-K_{\mathrm{s}}\right)=0.065 \times A_{\mathrm{V}}($ Chini \& Wargau 1998), and the standard gas-to-extinction ratio, $N_{\mathrm{H}_{2}} / A_{\mathrm{V}} \sim 1.25 \times 10^{21} \mathrm{~cm}^{-2}$ $\mathrm{mag}^{-1}$ (Dickman 1978). This estimated column density seems to be consistent with that estimated from the $\mathrm{CO}$ data, $\sim 1.3-4.1 \times$ $10^{22} \mathrm{~cm}^{-2}$. Adopting a width of $\sim 3$ '.0 $(\sim 1.4 \mathrm{pc})$ as the depth of Region 1, we can derive a number density of $n_{\mathrm{H}_{2}} \sim 6.5 \times$ $10^{3} \mathrm{~cm}^{-3}$. Since Region 2 is located around the symmetric axis of the cloud, the column density in Region 2 is expected to be close to the central/maximum column density if the local density is uniform. The average of color excess $E\left(H-K_{\mathrm{s}}\right)$ is $\sim 1.1 \pm 0.4 \mathrm{mag}$ for 41 sources toward Region 2, and $N_{\mathrm{H}_{2}} \sim 2.1 \times 10^{22} \mathrm{~cm}^{-2}$ is roughly obtained. This column density also seems to be consistent with that estimated from the CO data, $\lesssim 2.1 \times 10^{22} \mathrm{~cm}^{-2}$. Adopting the width of $\sim 8^{\prime} .0$ $(\sim 3.5 \mathrm{pc})$ as the depth of Region $2, n_{\mathrm{H}_{2}} \sim 2.0 \times 10^{3} \mathrm{~cm}^{-3}$ can be obtained in Region 2 . The estimated number density in Region 1 is $\sim 3-4$ times higher than that in Region 2, supporting the idea that Region 1 was strongly compressed by UV and then became higher in density. This result does not seem to match the simulation results for the nonmagnetized clouds that the number density in the tip is expected to be higher by more than one order of magnitude than that of the area away from the tip (e.g., Figures 3, 7, and 10 of Miao et al. 2009). However, the simulation taking into account the magnetic pressure predicts a smaller difference of a factor of $<10$ (e.g., Figure 8 of Miao et al. 2006), which is more consistent with our observations. Motoyama et al. (2013) also suggests flatter column density profiles in the magnetized cases than in the nonmagnetized ones.

We estimated the strengths of the magnetic fields $\left(B_{\|}\right)$ projected on the plane of the sky toward the two regions, using 


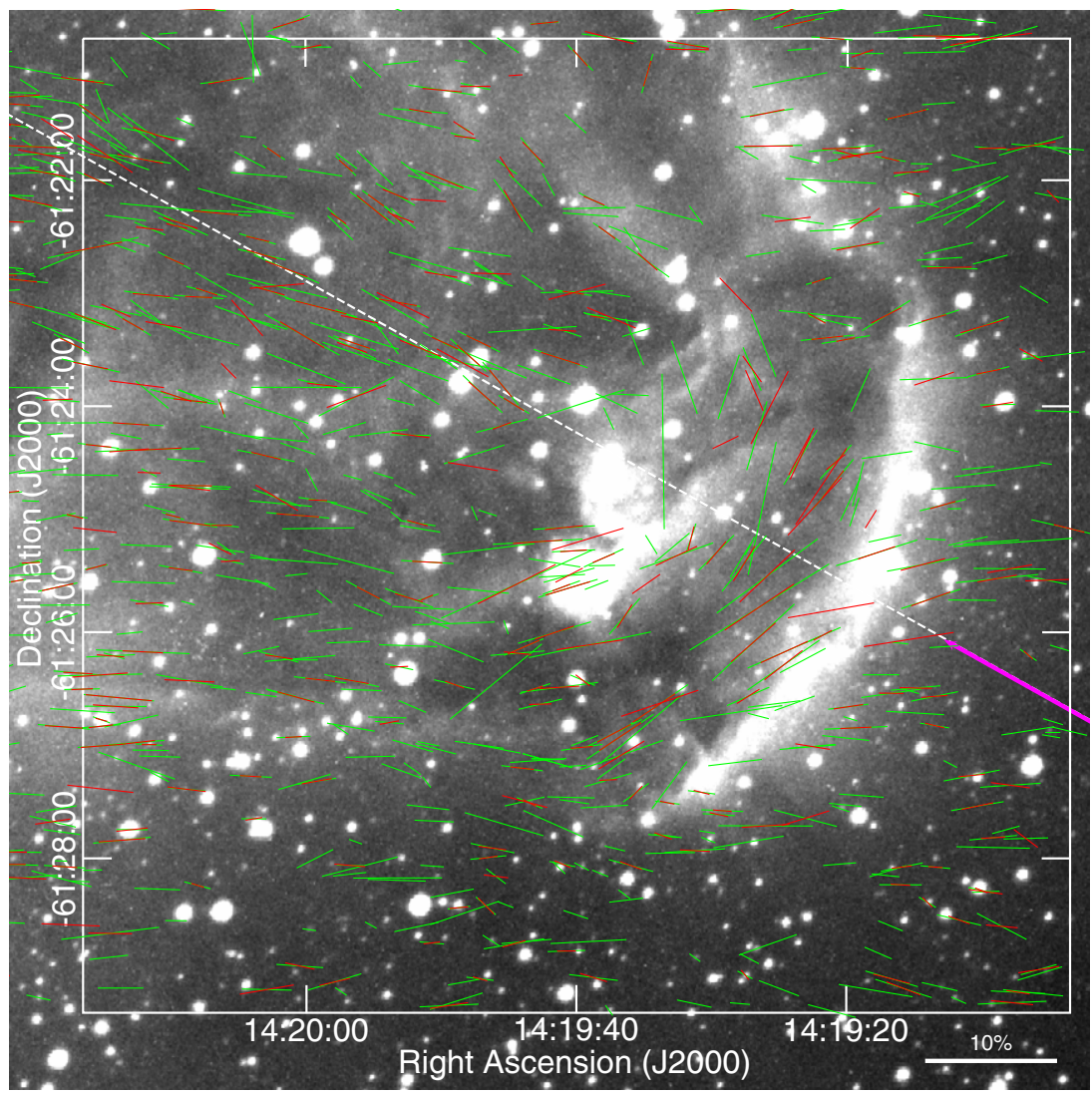

Figure 6. Polarization vector map superposed on the SuperCOSMOS $\mathrm{H}_{\alpha}$ image. $H$-and $K_{\mathrm{s}}$-band polarization vectors are shown with green and red vectors, respectively. A $10 \%$ vector is shown at the bottom right. The incident UV radiation direction is shown with a purple arrow, and its extended line (our adopted approximately symmetry axis) is shown by a white dashed line. Nearly all of the vector directions are consistent between two bands within their errors of $\leqslant 10^{\circ}$ for sources with polarization vectors at both bands.

the CF method,

$$
B_{\|}=Q \sqrt{4 \pi \rho} \frac{\sigma_{v}}{\sigma_{\theta}},
$$

where $\rho$ is the mean density of the cloud, $\sigma_{v}$ is the velocity dispersion, $\sigma_{\theta}$ is the dispersion of the polarization vector angles, and $Q \sim 0.5$ is a correction factor for $\sigma_{\theta} \lesssim 25^{\circ}$ (Ostriker et al. 2001). The magnetic field toward Region 1 seems to be slightly bent along the bright-rim tip, so we modeled the shape of the magnetic field with a parabolic function, assuming that the magnetic field direction is uniform along the tip rim. The magnetic field was adequately fitted with the slopes of the polarization vectors by rotating the parabolic function by $60^{\circ}$ in an easterly direction. We excluded sources having a large difference from the average. If the vectors are shown at two bands for a single source in Figure 6, only the single P.A. with less error was used for the fitting. At first, we calculated the dispersion $\left(\sigma_{\theta}\right)$ from the best-fit model for 23 sources in Region 1. Then, removing the dispersion due to the measurement uncertainties of the polarization angles, we obtained the corrected dispersion $\sigma_{\theta}^{\prime}$ of $\sim 11.4\left(\sigma_{\theta}^{\prime} \sim\right.$ $\sqrt{\sigma_{\theta}^{2}-\sum \Delta \theta^{2} / n}$ ) and used this for estimating the magnetic field strength with the $\mathrm{CF}$ method. The ${ }^{13} \mathrm{CO}$ velocity widths toward Region 1 are $\sim 1.3-1.5 \mathrm{~km} \mathrm{~s}^{-1}$ in Figure 8(c). Therefore, we adopted $1.4 \mathrm{~km} \mathrm{~s}^{-1}$ as a representative velocity width toward Region 1, which corresponds to the velocity dispersion $\left(\sigma_{v}\right)$ $\sim 0.64 \mathrm{~km} \mathrm{~s}^{-1}$. Adopting the molecular weight $\mu \sim 2.3$, we obtained $B_{\|} \sim 90 \mu \mathrm{G}$.
For Region 2, assuming that the direction of the magnetic field is uniform nearly along the symmetric axis, we obtained $\sigma_{\theta}^{\prime} \sim 16.4$ for 41 sources in Region 2 after removing the uncertainties. The $\mathrm{CO}$ observations only covered the southwest half of Region 2 . The ${ }^{13} \mathrm{CO}$ velocity width toward the southwest half of Region 2 appears to be $\sim 1.4-1.9 \mathrm{~km} \mathrm{~s}^{-1}$ and to decrease with distance away from the southwest edge of Region 2 along the symmetry axis. Therefore, we adopted the velocity width of $\sim 1.4 \mathrm{~km} \mathrm{~s}^{-1}$ near the center of Region 2 as a representative velocity width of Region 2 and obtained $B_{\|} \sim 30 \mu \mathrm{G}$.

It should be noted that the polarization vector maps toward SFO 74 might be contaminated by the polarized sources lying foreground and/or background to the cloud. However, this effect is probably small toward the dense parts of the cloud, i.e., Regions 1 and 2, because (1) the sources far behind the cloud, which have already undergone large interstellar absorption at the far side of the cloud, would not be detectable with our criteria, and (2) the extinction of foreground dwarfs, which are located at $H-K_{\mathrm{s}} \lesssim 0.2$ (Figure 3), is estimated to be $A_{V} \lesssim 1.5 \mathrm{mag}$, adopting $\sim 0.1 \mathrm{mag}$ as the intrinsic color $\left(H-K_{\mathrm{s}}\right)$ of the dwarfs and using the reddening law, $E\left(H-K_{\mathrm{s}}\right)=$ $0.065 \times A_{V}$ (Chini \& Wargau 1998). This extinction is much smaller than those of Regions 1 and $2\left(A_{V} \sim 23\right.$ and $\left.17 \mathrm{mag}\right)$. Therefore, the contamination from the foreground and/or background interstellar polarization is negligibly small in Regions 1 and 2 .

The magnetic field of Region 2 might be also enhanced by the convergence flow with respect to the original magnetic field, while that of Region 1 might be enhanced by the ionized front. 

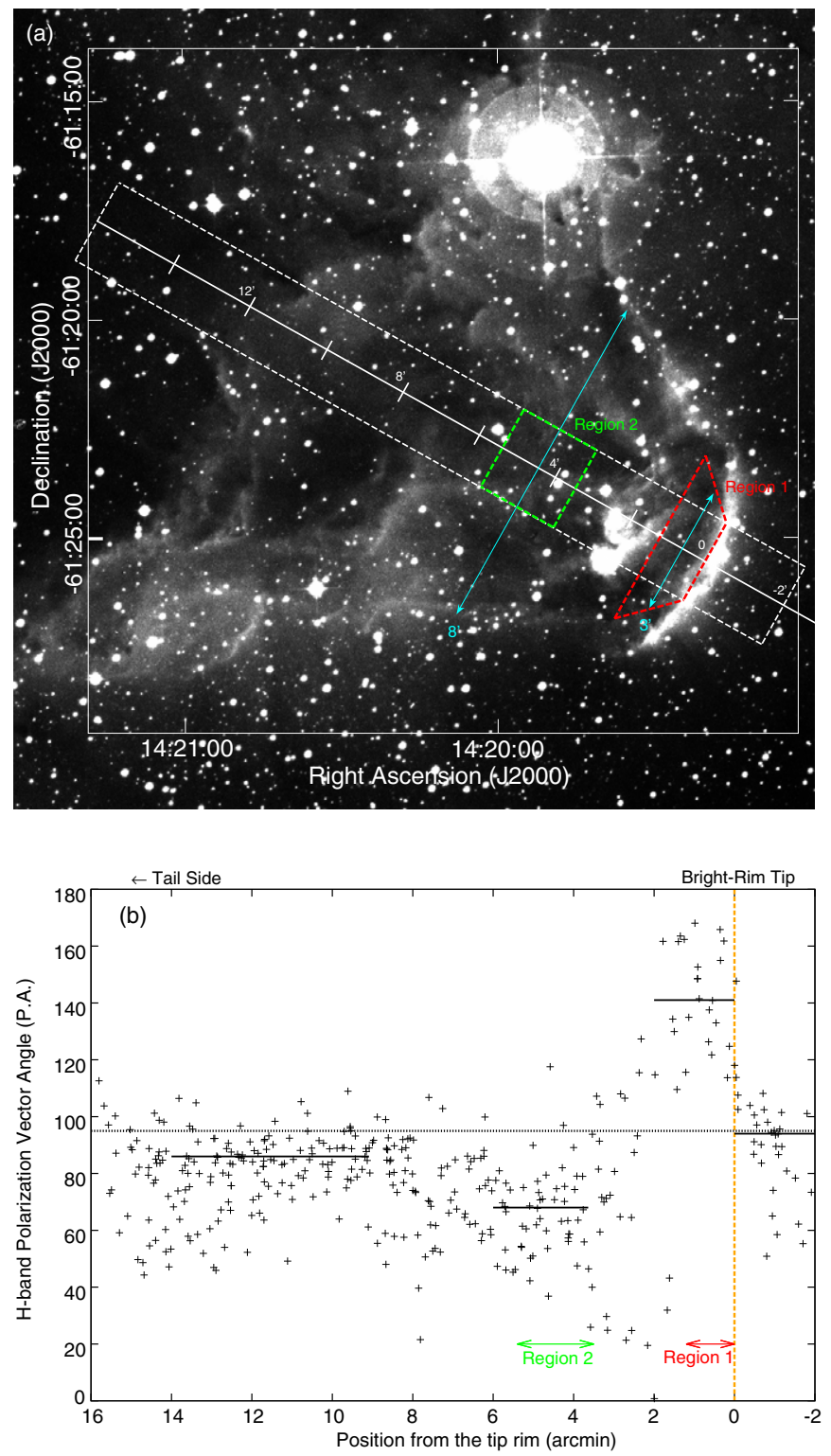

Figure 7. (a) Areas for analysis superposed on the SuperCOSMOS $\mathrm{H}_{\alpha}$ image. A white dashed box with a width of $2^{\prime}$ is the area that contains the sources used for plotting (b). Region 1 (red dashed trapezoid) and Region 2 (green dashed rectangle) are the areas that we used for analysis. Widths, which we adopted as the depths of two regions for analysis, are shown with cyan arrows. (b) Polarization angle of $H$-band vectors vs. position from the bright-rim tip. A dashed line shows the average of the ambient polarization direction. Solid lines show the averages of the polarization directions and the sample ranges (see Section 3.2).

The ratio of the magnetic field strength of Region 1 to that of Region 2 is derived to be $\gtrsim 2$, implying that the magnetic field of Region 1 has been more strongly enhanced. The $\mathrm{CF}$ method is considered to be a rough estimate of the magnetic field strength, and the derived strength might have a large uncertainty. However, we can still discuss the relative ratio of the derived magnetic field strengths because the physical parameters used were derived in the same way. The uncertainty in density is the largest, but we note that the magnetic field strength is proportional to the root of the density.

We compare the magnetic pressure, $P_{B}=B^{2} / 8 \pi$, with the turbulent pressure, $P_{\text {turb }}=\rho \sigma_{\text {turb }}^{2}$ in Region 1. $P_{B}$ is calculated to be $\sim 3.3 \times 10^{-10} \mathrm{dyn} \mathrm{cm}^{-2}$, adopting $B_{\|} \sim 90 \mu \mathrm{G}$ as $B$. This estimated value would be the lower limit because the magnetic field may not be running perpendicular to the line of the sight. Using a 1D velocity dispersion $\left(\sigma_{v}\right)$ of $\sim 0.64 \mathrm{~km} \mathrm{~s}^{-1}$, $P_{\text {turb }}$ is $\sim 3.1 \times 10^{-10} \mathrm{dyn}_{\mathrm{cm}^{-2}}$. These comparable estimated values imply that the magnetic pressure significantly contributes to the internal pressure and that the total pressure is $\sim 1.0 \times$ $10^{-9} \mathrm{dyn} \mathrm{cm}^{-2}$. For Region 2, we obtained $P_{B} \sim 4.7 \times$

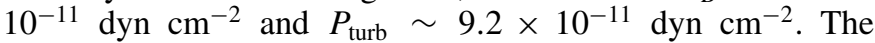
magnetic pressure is estimated to be slightly smaller than the turbulent pressure in Region 2.

Then, we compare the total internal pressure in Region 1 with the external pressure, i.e., the radiation pressure $P_{\text {rad }}$ and the post-shock pressure by the ionization front. The maximum ionizing flux is $F=4.5 \times 10^{8} \mathrm{~cm}^{-2} \mathrm{~s}^{-1}$, and the energy of an ionizing photon $h v \geqslant 13.6 \mathrm{eV}$. If we assume the maximum average energy of an ionizing photon $h v_{\max }=20 \mathrm{eV}$ (in fact, it is much lower than that), then we can find the upper limit of $P_{\text {rad }}=F / c<4.8 \times 10^{-13} \mathrm{dyn} \mathrm{cm}^{-2}$. It is seen that $P_{\text {rad }}$ is negligible. To estimate the post-shock pressure, we assumed plausible physical quantities of $n \sim$ a few $\times 10^{3} \mathrm{~cm}^{-3}$ and $T \sim 8000 \mathrm{~K}$ for the number density and the temperature of the ionization front, respectively. We thus derived the external pressure of a few $\times 10^{-9}$ dyn $\mathrm{cm}^{-2}$. This value is comparable to or slightly larger than the total internal pressure, and thus these two regions may be in an equilibrium state or in a weak overpressure state of the ionization front. If this is the case, we suggest that the magnetic field plays an important role in the pressure balance between these two regions and in the evolution of Cloud A.

The dynamical state of a magnetized cloud is measured by the ratio between the cloud mass $\left(M_{\text {cloud }}\right)$ and the magnetic flux $(\Psi)$, i.e., the mass-to-flux ratio, which is given by $M_{\text {cloud }} / \Psi=\mu m_{\mathrm{H}} N_{\mathrm{H}_{2}} / B \sim 2.0 \times\left(M_{\text {cloud }} / \Psi\right)_{\text {critical }}$ for Region 1, where $\left(M_{\text {cloud }} / \Psi\right)_{\text {critical }}$ is the critical value for magnetic stability of the cloud $\left(=\left(4 \pi^{2} G\right)^{-1 / 2} ;\right.$ Nakano \& Nakamura 1978). Here, we assume that the magnetic field is almost perpendicular to the line of sight, but that is not necessarily so. Therefore, the actual magnetic field strength might be larger, and the net massto-flux ratios could be close to the critical value. This implies that the magnetic field is likely to play an important role in the cloud dynamics, such as the evolutional timescale of the cloud, the density and the thickness of the compressed layer, and thus star formation in Cloud A. For Region 2, $M_{\text {cloud }} / \Psi$ is given by $\sim 3.8 \times\left(M_{\text {cloud }} / \Psi\right)_{\text {critical }}$, which is larger by a factor of $\sim 2$ than that in Region 1, and the turbulent pressure is dominant.

\subsection{Flat-topped Shape}

As seen in Figure 4, the bright rim of SFO 74 appears to consist of two parts: the tip facing the exciting star and the wings extending toward both tail sides (Figure 12). We briefly consider this flat-topped shape.

The magnetic fields just behind the wing rims seem to run along the bright rims like that just behind the tip rim (Figure 4). The strength of the magnetic field just behind the tip, however, could be larger than that just behind the wing. The layer just behind the tip rim is perpendicular to the incident UV direction and might be more powerfully compressed by the UV radiation than that just behind the wing rims, which are nearly parallel or oblique to the UV radiation. We speculate that this flat-topped shape is formed by the larger increase of the magnetic field strength just behind the tip.

As for the physical explanation of the flat-topped shape of SFO 74, we look at the magnetohydrodynamic equation of motion, which is one of the four astrophysical fluid 

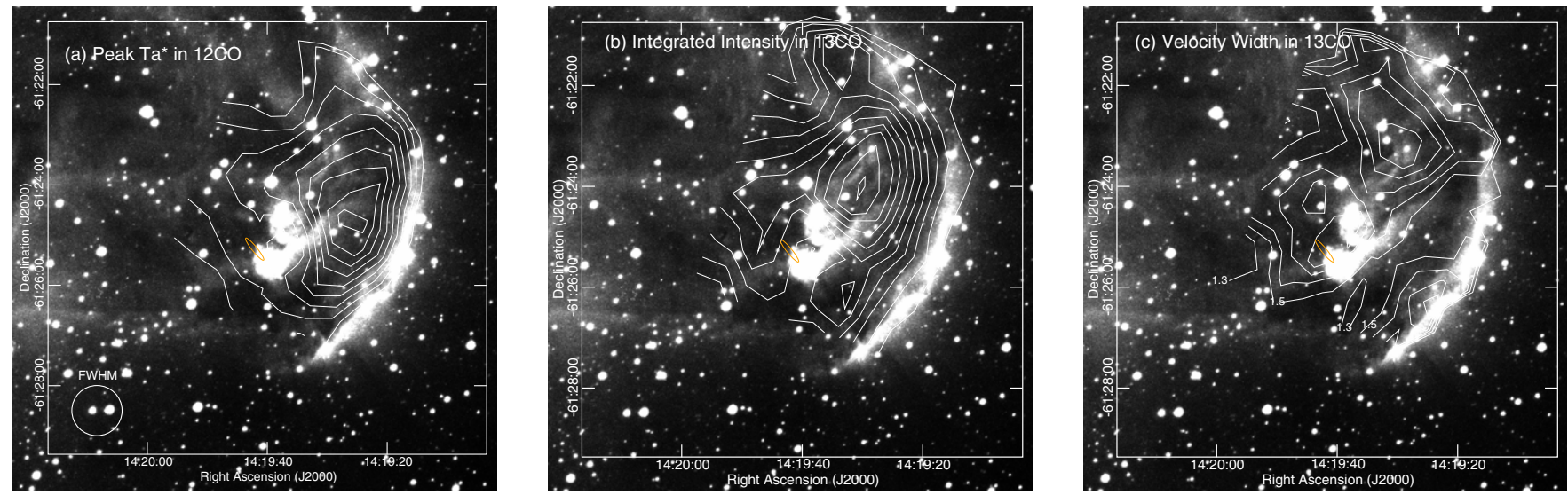

Figure 8. (a) Peak $T_{\mathrm{A}}^{*}$ map of ${ }^{12} \mathrm{CO}(1-0)$ superposed on the SuperCOSMOS $\mathrm{H} \alpha$ image. The lowest contour level is $10 \mathrm{~K}$, and the contour interval is $2 \mathrm{~K}$ in $T_{\mathrm{A}}^{*}$. The beam size is shown in the lower left corner. (b) Integrated intensity map of ${ }^{13} \mathrm{CO}(1-0)$ superposed on the SuperCOSMOS H $\alpha$ image. The velocity range is -31.4 to $-25.9 \mathrm{~km} \mathrm{~s}^{-1}$ in $v_{\mathrm{LSR}}$. The lowest contour level is the $6 \sigma$ level $\left(=1.04 \mathrm{~K} \mathrm{~km} \mathrm{~s}^{-1}\right.$ in $\left.T_{\mathrm{A}}^{*}\right)$, and the contour interval is $6 \sigma$. (c) Velocity width map of ${ }^{13} \mathrm{CO}$. The lowest contour level is $1.3 \mathrm{~km} \mathrm{~s}^{-1}$, and the contour interval is $0.2 \mathrm{~km} \mathrm{~s}^{-1}$. The position of IRAS 14159-6111 is shown with an orange error ellipse of position.

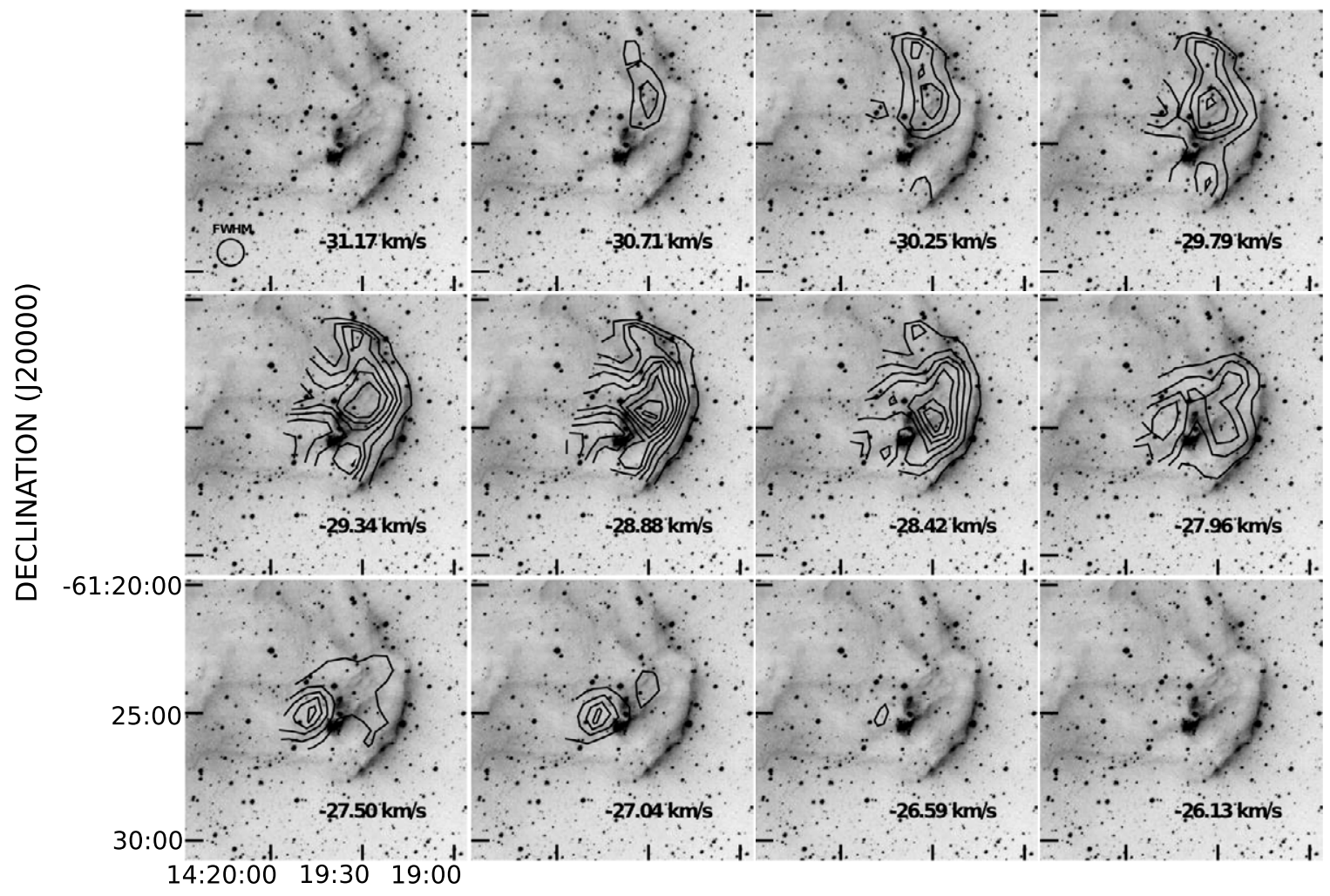

RIGHT ASCENSION (J2000)

Figure 9. Channel maps of ${ }^{13} \mathrm{CO}$ superposed on the SuperCOSMOS $\mathrm{H}_{\alpha}$ image. The center LSR velocity of each channel is shown on the bottom right of each panel. The lowest contour level is the $6 \sigma$ level $\left(=0.3 \mathrm{~K} \mathrm{~km} \mathrm{~s}^{-1}\right.$ in $\left.T_{\mathrm{A}}^{*}\right)$, and the contour interval is $6 \sigma$. The beam size is shown in the lower left corner in the panel of $v_{\mathrm{LSR}}=-31.17 \mathrm{~km} \mathrm{~s}^{-1}$

equations,

$$
\rho \frac{d \mathbf{V}}{d t}=-\nabla p+\nabla \cdot \mathbf{T}
$$

where all of symbols are of their conventional physical meaning, and the magnetic pressure tensor $T_{\mathrm{ij}}=\left(B_{i} B_{j}-\delta_{i j} B^{2} / 2 / 4 \pi\right)$ $(i, j=1,2,3)$.

According to Figure 7(b), the average magnetic field direction behind the tip has a P.A. of $\sim 68^{\circ}$, approximately parallel to the central symmetric line of SFO 74 and the UV radiation direction $\left(\sim 60^{\circ}\right)$. If we choose a $z$-axis parallel to the direction of the average magnetic field and UV radiation and assume the field just behind the compressed surface layer of SFO 74, the above equation becomes

$$
\rho \frac{d \mathbf{V}}{d t}=-\nabla \cdot \mathbf{P},
$$

where the deduced pressure tensor

$$
\mathbf{P}=\left(\begin{array}{ccc}
p+\frac{B^{2}}{8 \pi} & 0 & 0 \\
0 & p+\frac{B^{2}}{8 \pi} & 0 \\
0 & 0 & p-\frac{B^{2}}{8 \pi}
\end{array}\right)
$$



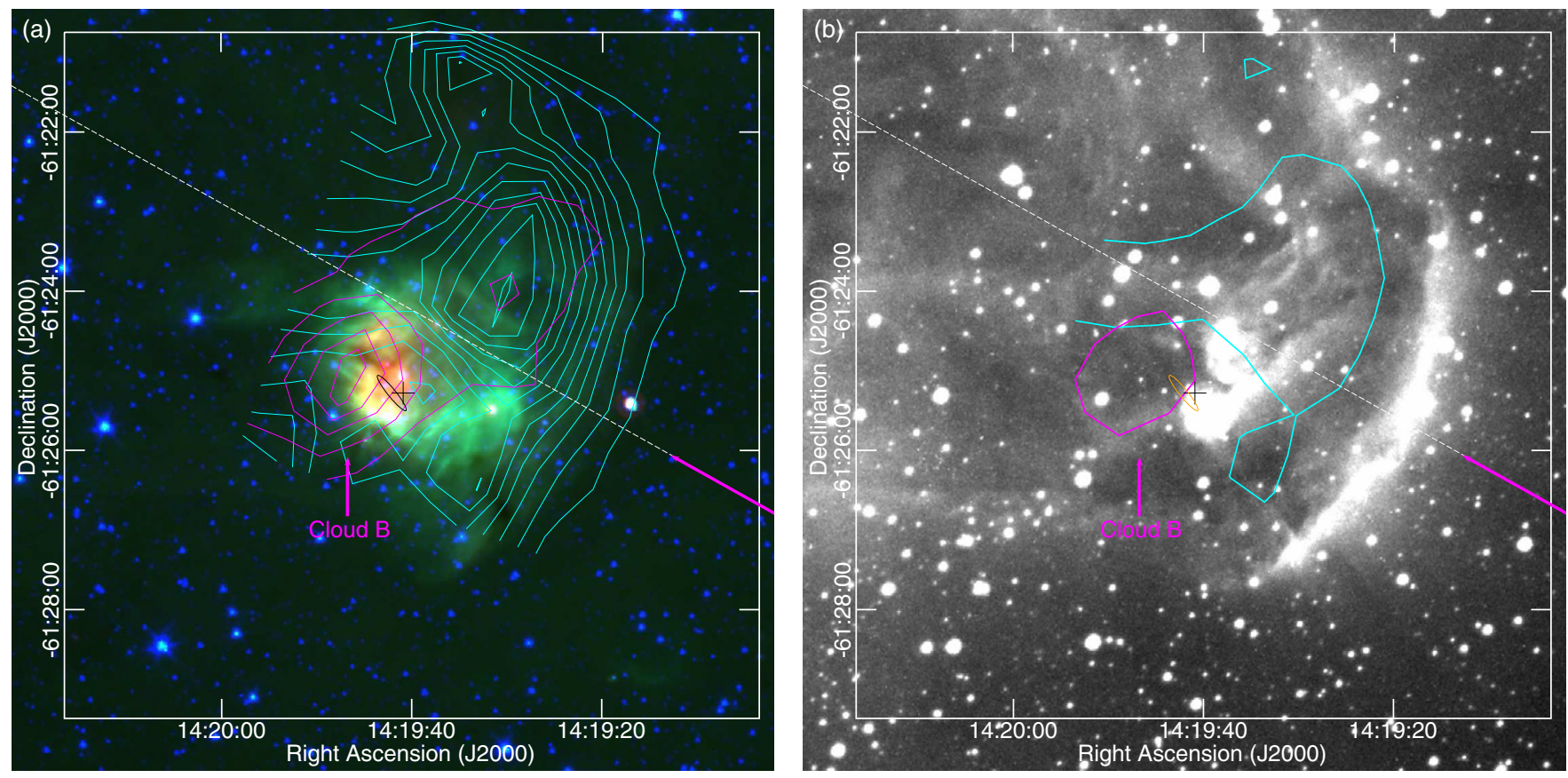

Figure 10. (a) Integrated intensity maps of Cloud A (cyan; $v_{\mathrm{LSR}}=-31.35$ to $-27.78 \mathrm{~km} \mathrm{~s}^{-1}$ ) and of Cloud B (magenta; $v_{\mathrm{LSR}}=-27.69$ to $-25.94 \mathrm{~km} \mathrm{~s}{ }^{-1}$ ) superposed on a three-color composite image, which has been constructed from the Spitzer-GLIMPSE $3.5 \mu \mathrm{m}$ (blue) image, $8 \mu \mathrm{m}$ (green) image (Benjamin et al. 2003), and Spitzer-MIPSGAL $24 \mu \mathrm{m}$ (red) image (Carey et al. 2009). The lowest contour level for Cloud A is the $6 \sigma$ level (= $0.85 \mathrm{~K} \mathrm{~km} \mathrm{~s}{ }^{-1}$ in $\left.T_{\mathrm{A}}^{*}\right)$, and the contour interval is $6 \sigma$. The lowest contour level for Cloud B is the $6 \sigma$ level $\left(=0.6 \mathrm{~K} \mathrm{~km} \mathrm{~s}^{-1}\right.$ in $\left.T_{\mathrm{A}}^{*}\right)$, and the contour interval is $6 \sigma$. (b) Cloud A and B positions superposed on the Super COSMOS $\mathrm{H}_{\alpha}$ image. The solid lines show $50 \%$ of the peak integrated intensity in ${ }^{13} \mathrm{CO}$. The cyan line is for Cloud A, and the magenta one is for Cloud B. The position of IRAS 14159-6111 is shown with a black or orange error ellipse of position. The peak position of radio continuum with ACTA (Thompson et al. 2004) is shown by a black cross. The symmetric axis is shown by a white dashed line.

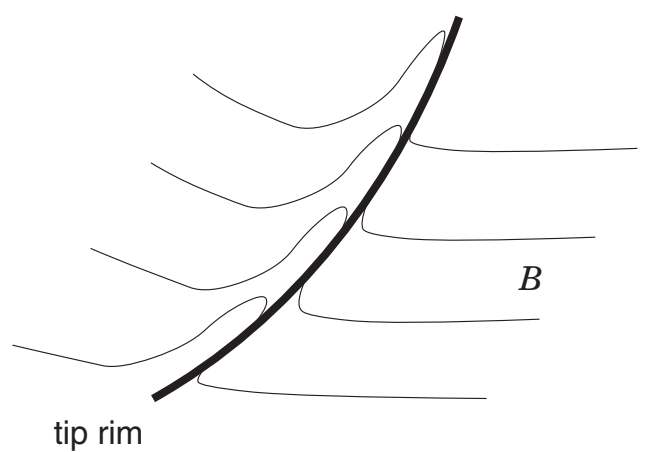

Figure 11. Schematic drawing of the magnetic field just behind the brightrim tip.

We can see that the magnetic field increases the cloud pressure by an amount of $\left(B^{2} / 8 \pi\right)$ in the elongation direction perpendicular to the magnetic field ( $x$ and $y$ ) but decreases the cloud pressure by the same amount in the parallel direction (z) (Fitzpatrick 2014). Therefore, we can expect that the $z$-axis directional magnetic field B in SFO 74 makes the structure of the cloud stretch in the width direction perpendicular to the magnetic field line and contract in the parallel length direction. This effect further quashes the magnetic field line loops created by UV-radiation-induced shock, so that the loop lines appear to be around the bright rim. The strength of this magnetic effect increases with the local density owing to a positive dependence of magnetic field B on number density. Therefore, a flatter bright tip rim than that in the zero magnetic field case appears on the star-facing side of SFO 74.

Although our main focus in this paper is to investigate the physical properties of the head part of SFO 74, we are also

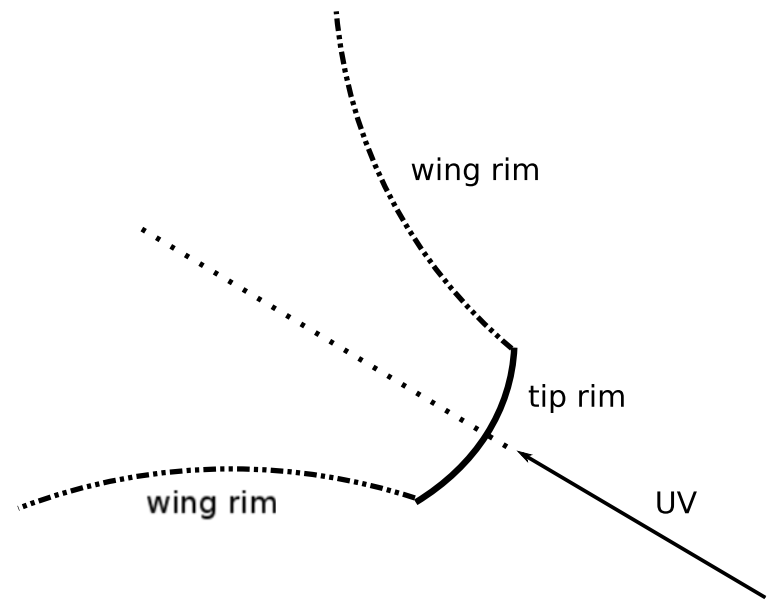

Figure 12. Schematic drawing that shows the flat-topped shape of SFO 74. The bright rim seems to consist of two parts: tip rim and wing rims.

interested in deriving a reasonable picture of the development of the morphology of the two-wing rim structure shown in Figure 12. As we analyzed above, the magnetic field line behind the Region 1 part of the type B SFO 74 is much less affected by the shock, and an increase of the magnetic pressure along the $x-y$ direction expands the width of the tail section (Region 2) more and more efficiently toward the rear end of SFO 74 than in the head part, so that we see the gradually outward-curved wing rim and the magnetic field lines naturally parallel to the wing rim. However, the wildly curved-out part at the rear end of the wing rim might not be caused by the expansion effect of the $z$-directional magnetic field only, which might also be the result of the Rocket effect induced by the photoevaporation of 
Table 1

Candidates for Exciting Stars of Ultracompact H II Region

\begin{tabular}{|c|c|c|c|c|c|c|c|c|}
\hline $\begin{array}{l}\text { Near-IR } \\
\text { Source }\end{array}$ & R.A.(J2000) & Decl.(J2000) & $\begin{array}{c}J \\
(\mathrm{mag})\end{array}$ & $\begin{array}{c}H \\
(\mathrm{mag})\end{array}$ & $\begin{array}{c}K_{\mathrm{S}} \\
(\mathrm{mag})\end{array}$ & $\begin{array}{c}\text { Optical } \\
\text { Counterpart }\end{array}$ & $\begin{array}{l}\text { Near-IR } \\
\text { Sp. Type }\end{array}$ & $\begin{array}{c}\text { Nebula } \\
\text { Association }\end{array}$ \\
\hline 1 & $14^{\mathrm{h}} 19^{\mathrm{m}} 42^{\mathrm{s}} .87$ & $-61^{\circ} 25^{\prime} 12^{\prime \prime}$ & $19.25 \pm 0.35$ & $14.09 \pm 0.04$ & $11.61 \pm 0.02$ & No & B1 & Yes \\
\hline 2 & $14^{\mathrm{h}} 19^{\mathrm{m}} 40^{\mathrm{s}} .92$ & $-61^{\circ} 25^{\prime} 05^{\prime \prime}$ & $12.76 \pm 0.04$ & $11.16 \pm 0.04$ & $10.28 \pm 0.02$ & No & B2 & Yes? \\
\hline 3 & $14^{\mathrm{h}} 19^{\mathrm{m}} 44^{\mathrm{s}} .42$ & $-61^{\circ} 25^{\prime} 35^{\prime \prime}$ & $19.07 \pm 0.19$ & $14.58 \pm 0.04$ & $12.16 \pm 0.02$ & No & B2 & Yes \\
\hline 4 & $14^{\mathrm{h}} 19^{\mathrm{m}} 48^{\mathrm{s}} .23$ & $-61^{\circ} 24^{\prime} 57^{\prime \prime}$ & $12.04 \pm 0.04$ & $10.68 \pm 0.04$ & $9.96 \pm 0.02$ & Yes & $\mathrm{M}$ & $\overline{\text { Foreground }}$ \\
\hline 5 & $14^{\mathrm{h}} 19^{\mathrm{m}} 36^{\mathrm{s}} .44$ & $-61^{\circ} 25^{\prime} 52^{\prime \prime}$ & $14.18 \pm 0.04$ & $11.56 \pm 0.04$ & $10.31 \pm 0.02$ & No & Giant & Background \\
\hline
\end{tabular}

the ionized gas (Oort \& Spitzer 1995). In order to reveal the actually morphological and physical evolution of SFO 74, we are preparing a 3D simulation by fully treating the magnetic field (J. Miao et al. 2015, in preparation).

\subsection{Star Formation Activity}

\subsubsection{Cloud A}

As shown in Figure 10, the peak position of the radio continuum with ACTA (Thompson et al. 2004) and the position of the IRAS/Midcourse Space Experiment (MSX) point source are not located toward Cloud A, but toward Cloud B. We did not see any sign of high-mass star formation from the Spitzer composite image (Figure $10(\mathrm{a})$ ) or of ${ }^{12} \mathrm{CO}$ molecular outflow from the Mopra data. Thus, the star formation in Cloud A may not be currently active. The $8 \mu \mathrm{m}$ emission is considered to be dominated by polycyclic aromatic hydrocarbons (PAHs) and useful for identifying a cavity wall of an $\mathrm{H}$ II region (e.g., Rathborne et al. 2002; Urquhart et al. 2003, 2009). No strong $8 \mu \mathrm{m}$ emission is associated with Cloud A (see green color in Figure 10(a)), and the ${ }^{12} \mathrm{CO}$ data suggest external heating from the exciting star of RCW 85. Thus, we concluded that Cloud A was not strongly affected by the UC $\mathrm{H}$ II region.

\subsubsection{Cloud B}

In contrast to Cloud A, Cloud B has some signs of active star formation. Hence, we examine more closely star formation in Cloud B.

The exciting star of the UC H II region is assigned to be a B1type star from the radio flux, although it is unresolved spatially. In order to identify the exciting stars, we try to search for their NIR counterparts within $60^{\prime \prime}$ of the IRAS position, the UC H II region and the brightest part of the $8 \mu \mathrm{m}$ PAH emission. In the $H$ versus $H-K_{\mathrm{s}}$ diagram (Figure 13(a)), we found five candidates responsible for the $\mathrm{UC} \mathrm{H}$ II region lying above the reddening line of B2. We also made the color-color diagram for these sources (Figure 13(b)). These five sources are also marked on the threecolor-composite and monochrome images (Figures 13(c) and (d)), and their properties are presented in Table 1. Sources 4 and 5 are located outside of the mid-IR nebula, while sources 1,2 , and 3 lie within the brightest part of the nebula. Sources 1,2 , and 3 are likely to be embedded B1, B2, and B2 stars that suffer extinction as large as $A_{V} \sim 40, \sim 15$, and $\sim 40 \mathrm{mag}$, respectively (Figures 13(a) and (b)). The position of source 1 coincides quite well with that of the IRAS source. Therefore, source 1 is the most likely candidate responsible for current star formation activities such as the UC H II region, PAH emission, and far- to mid-infrared radiation.

In our NIR image (Figure 1(b)), a small stellar aggregation can be seen toward Cloud B. The center of this aggregation is only $\sim 20^{\prime \prime}$ west of the IRAS position, and several stars of this aggregation indicate color excess on the NIR color-color diagram. It is likely that this is a small stellar cluster associated with the exciting star mentioned above (e.g., Testi et al. 1998).

\section{SUMMARY}

We made the NIR imaging polarimetry toward SFO 74 . We found that the magnetic field runs along the bright rim in the layer just behind the bright rim, and that the whole magnetic field configuration, as well as the whole shape, is roughly symmetric with respect to the symmetric axis of the cloud. This configuration is most likely due to the UV radiation from the exciting star. We estimated the magnetic field strength of $\sim 90 \mu \mathrm{G}$ toward Region 1 (the tip region just behind the brightrim tip) and $\sim 30 \mu \mathrm{G}$ toward Region 2 (the inside region on the cloud axis), implying that the magnetic field strength toward the compressed layer (Region 1) has been more enhanced by the UV radiation than Region 2 . The magnetic pressure is roughly comparable to the turbulent pressure, and thus the magnetic field seems to make a significant contribution to the internal pressure in Region 1. The mass-to-flux ratio is close to the critical value in Region 1, indicating the importance of the magnetic field in the dynamics here. We speculate that the flat-topped shape of SFO 74 is due to the increase of the magnetic field strength just behind the tip. Since the number of observations that show clear magnetic field structures is still small, it is very important to make more polarimetric observations of BRCs and to conduct 3D MHD simulations in order to understand the magnetic field effect on the evolution of BRCs.

We are grateful for the support of the staff of SAAO during the observation runs. We also thank the anonymous referee for improving the manuscript. This work was partly supported by Grants-in-Aid for Scientific Research (24340038 and 24540233) from the Ministry of Education, Culture, Sports, Science, and Technology of Japan. T.K. and K.S. thank Y. Nakajima for assisting in the data reduction with the SIRPOL pipeline package. T.K. is a Research Fellow of the Japan Society for the Promotion of Science. M.T. is partly supported by JSPS Grants-in-Aid for Scientific Research.

\section{APPENDIX A}

\section{COMPARISON OF THE POLARIZATION OBSERVATIONS IN 2013 AND 2014}

We observed the northeast region of SFO 74 in 2014 with SIRPOL, in addition to the observation in 2013, because the total exposure time of 2013 (600 s per wave-plate angle) toward this region was shorter than those (900 s) toward the other regions. Therefore, we had a chance to examine the 

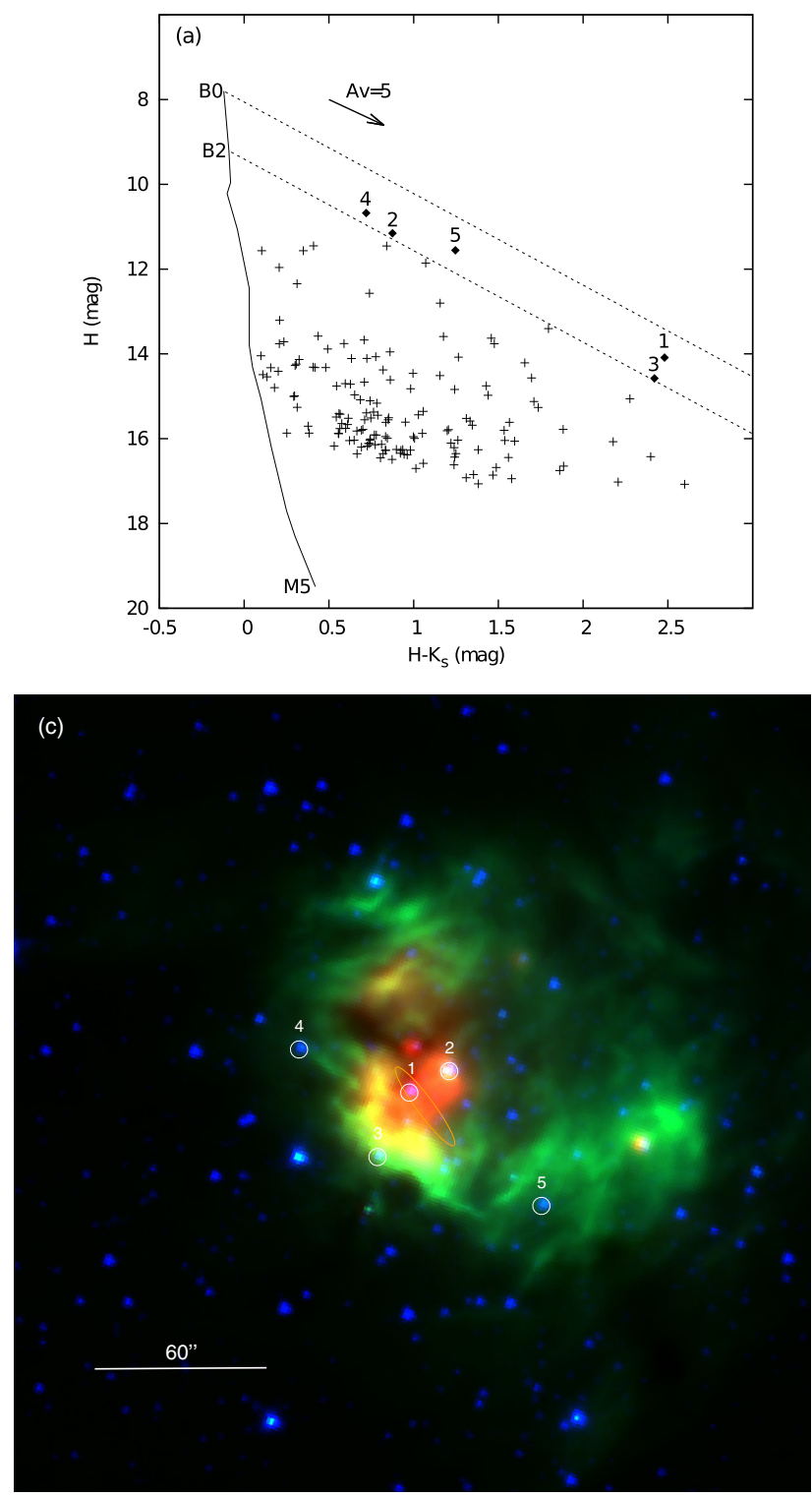
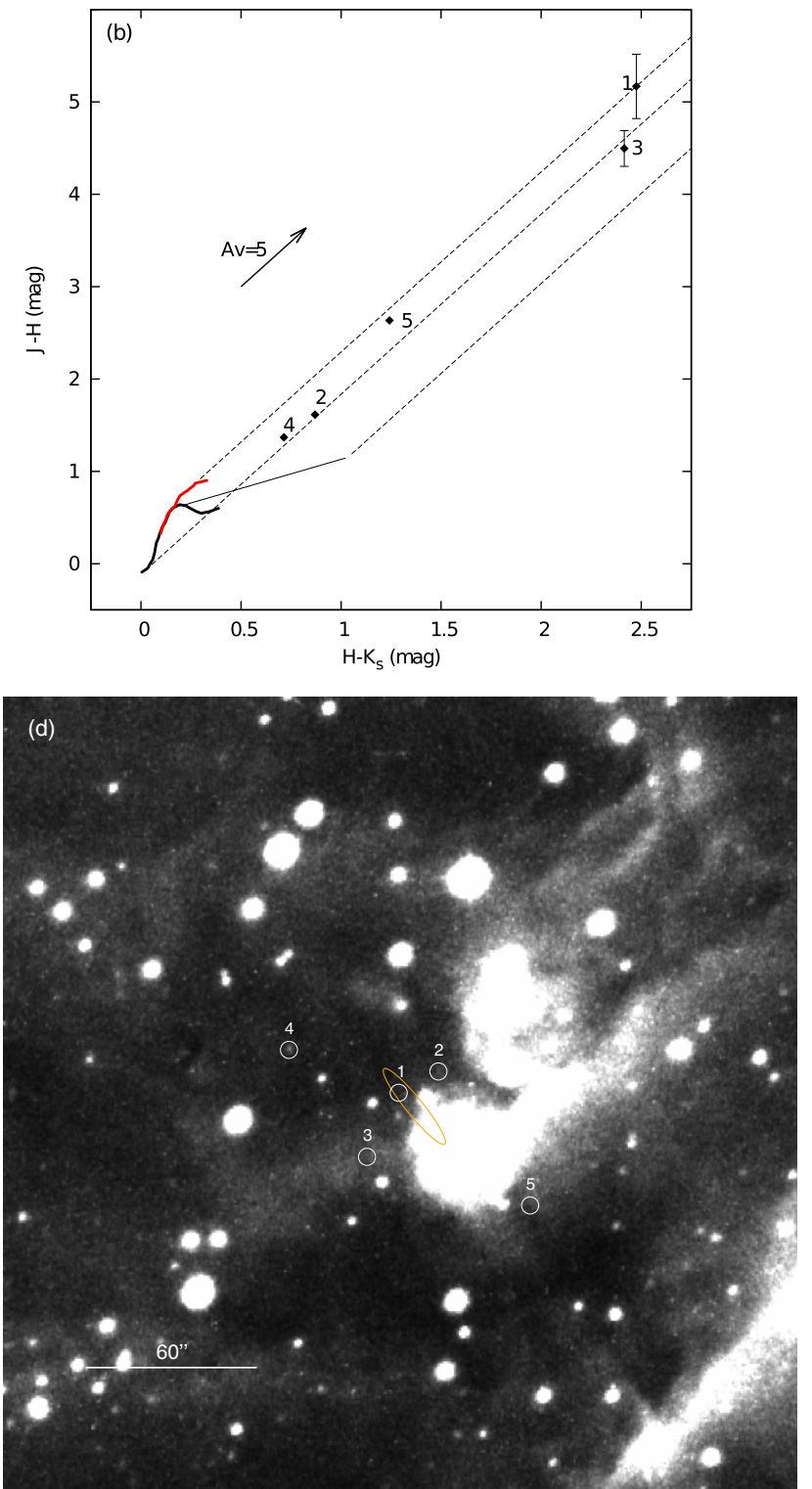

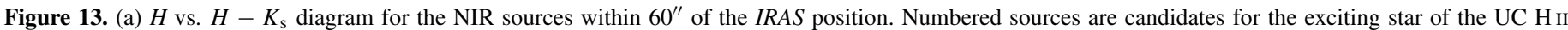

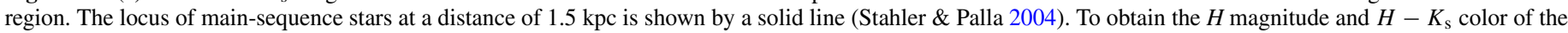

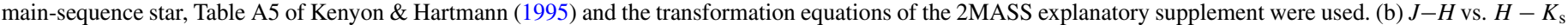

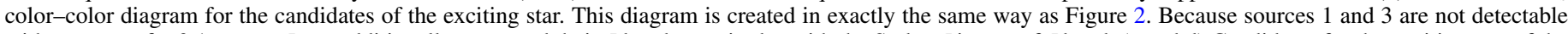

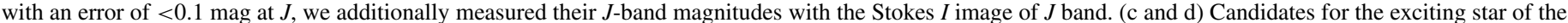

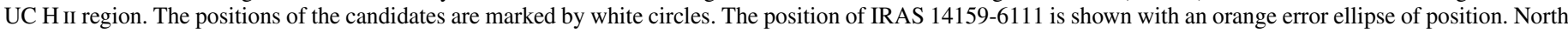

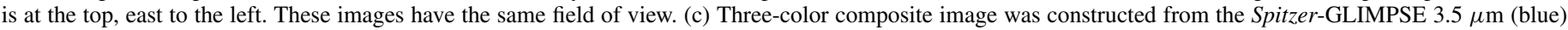
image, $8 \mu \mathrm{m}$ (green) image, and Spitzer-MIPSGAL $24 \mu \mathrm{m}$ (red) image, and (d) the monochrome image was taken from the Super COSMOS $\mathrm{H}_{\alpha}$ image.

consistency of the polarization measurements with SIRPOL between two years.

Figure 14 shows the $H$-band vector maps of 2013 and 2014 superposed on the $H$-band $I$ image obtained in 2014. We included only the sources that are detected, in both 2013 and 2014 , with $P / \Delta P>3.0\left(\Delta \theta \lesssim 10^{\circ}\right)$ in the range between the upper and lower limits of Figure 3 . Red and green vectors indicate those obtained in 2013 and 2014, respectively. Most of the green vectors (2014) are likely to be consistent with the red vectors (2013). Figure 15 shows the difference of polarization angles for each source between 2013 and 2014, where the horizontal axis is the root sum square of the polarization angle errors of 2013 and $2014\left(\Delta \theta_{\mathrm{RSS}}=\sqrt{\Delta \theta_{2013}^{2}+\Delta \theta_{2014}^{2}}\right)$. It is found that $62 \%$ of the sources have a difference of $<\Delta \theta_{\mathrm{RSS}}$ and that $88 \%$ of them have that of $<2 \Delta \theta_{\mathrm{RSS}}$. As the result of the $J$ and $K_{\mathrm{s}}$-band analysis with the same criteria, $46 \%$ and $58 \%$ of the sources have a difference of $<\Delta \theta_{\mathrm{RSS}}$, respectively. These results support the above consistency seen in Figure 14. We also examined the consistency of the polarization degree in $H$ band and found that $60 \%$ of the sources have a difference of the polarization degree of $<\sqrt{\Delta P_{2013}^{2}+\Delta P_{2014}^{2}}$ and that $88 \%$ of them have that of $<2 \sqrt{\Delta P_{2013}^{2}+\Delta P_{2014}^{2}}$. For $J$ and $K_{\mathrm{s}}$ bands, $61 \%$ and $55 \%$ of the sources have a difference of $<\sqrt{\Delta P_{2013}^{2}+\Delta P_{2014}^{2}}$, respectively. Therefore, we concluded that the polarization measurements with SIRPOL are consistent between two years. 


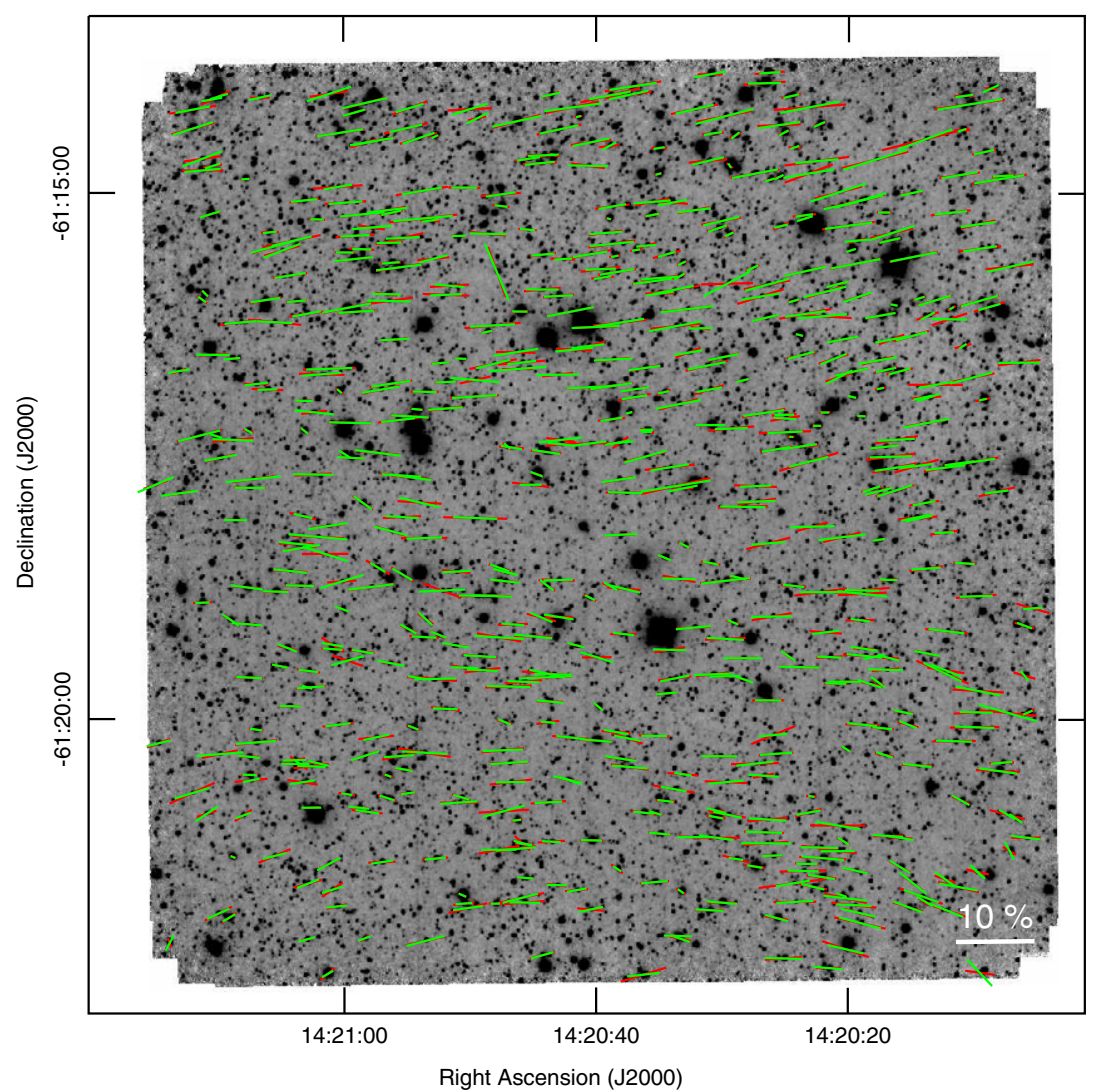

Figure 14. Polarization vector maps superposed on our $H$-band Stokes $I$ image in 2014. Red and green polarization vectors indicate ones obtained in 2013 and 2014 , respectively. A $10 \%$ vector is shown at the bottom right.

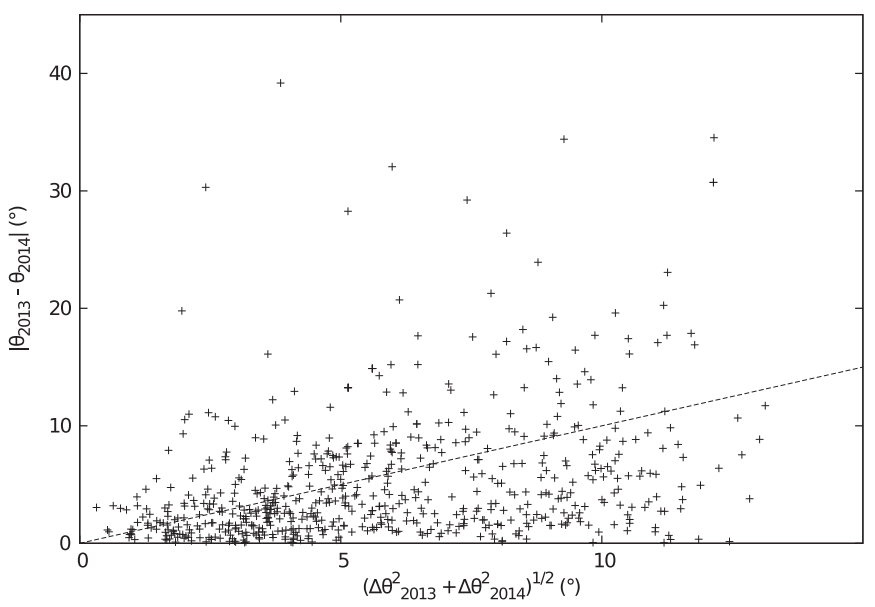

Figure 15. Difference of the polarization angles between 2013 and 2014 vs the root sum square of the polarization angle errors in 2013 and 2014 for the vectors shown in Figure 14. A dashed line shows a line of $\left|\theta_{2013}-\theta_{2014}\right|=$ $\sqrt{\Delta \theta_{2013}^{2}+\Delta \theta_{2014}^{2}}$.

\section{APPENDIX B}

\section{TRANSFORMATION ANGLE TO THE EQUATORIAL COORDINATE SYSTEM}

We had a chance to obtain polarimetric data of the $\mathrm{R}$ Mon nebula (e.g., Minchin et al. 1991), which is known as the object having a centrosymmetric vector pattern owing to the scattered light in the outer area of the nebula. This object enables us to examine the transformation angle from the SIRPOL coordinate system to the equatorial coordinate system. The SIRIUS camera is a stationary instrument, and SIRPOL is attached to SIRIUS. Because the polarimeter of SIRPOL is designed to be attached always in the same way, with the slide guide settled on the SIRIUS-side flange (Kandori et al. 2006), the transformation angle is considered to be highly reproducible. With these data, however, we tried to make sure that the transformation angle of $105^{\circ}$ with an accuracy of $\lesssim 3^{\circ}$ (Kandori et al. 2006) is applicable at present.

With SIRPOL, the $J H K_{\mathrm{s}}$ polarimetric observation of the $\mathrm{R}$ Mon nebula was made on 2014 March 29. We obtained 10 dithered observations, each $10 \mathrm{~s}$ long, at four wave-plate angles as one set of observations, and we repeated this six times. Thus, the total on-target exposure time was $600 \mathrm{~s}$ per each plate wave-plate angle. Sky images were also obtained between target observations and were used for medium sky subtraction. Twilight flat-field images were obtained at the beginning and/or end of the observations. R Mon is located in the peak of the cometary nebula NGC 2261 and is known as the source illuminating the nebula (Minchin et al. 1991), and we determined the coordinate of $\mathrm{R}$ Mon as a peak position from our $J$-band Stokes $I$ image, (R.A., decl. $)_{\mathrm{J} 2000}=\left(6^{\mathrm{h}} 39^{\mathrm{m}} 10^{\mathrm{s}} .0,+8^{\circ} 44^{\prime} 09^{\prime \prime} \cdot 7\right)$.

Figure 16 shows the $H$-band polarization vector map of R Mon superposed on the $H$-band Stokes $I$ image. Here we can see a centrosymmetric vector pattern around R Mon. As indicated in Figure 17, if the transformation angle is appropriate, the terminal points of the normal vectors to the polarization vectors ideally coincide with the illuminating source. In practice, however, they 


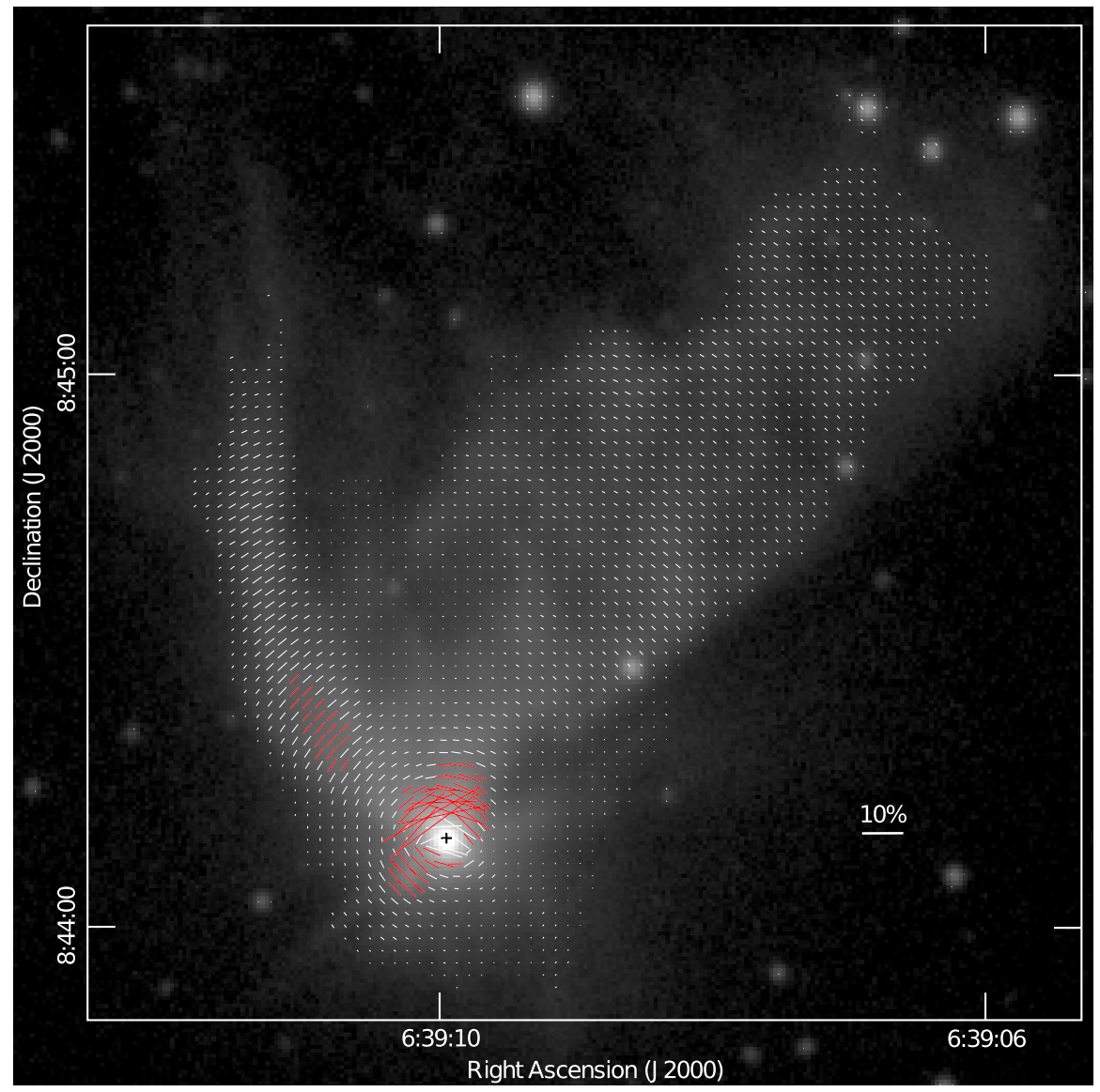

Figure 16. $H$-band polarization vector map of the R Mon nebula superposed on the $H$-band Stokes $I$ image with a logarithmic scale. A $10 \%$ vector is shown in the lower right corner. The vectors were made by $3 \times 3$ pixel binning. A black cross represents the position of R Mon. All vectors are shown in the region where the nebula intensity is greater than $10 \sigma$ above the mean sky level. The vectors with a polarization degree greater than $3 \%$ are indicated in red and were used to examine the transformation angle from the SIRPOL coordinate system to the equatorial coordinate system, excluding the vectors within a radius of $2^{\prime \prime}$ from the position of R Mon.

(a)

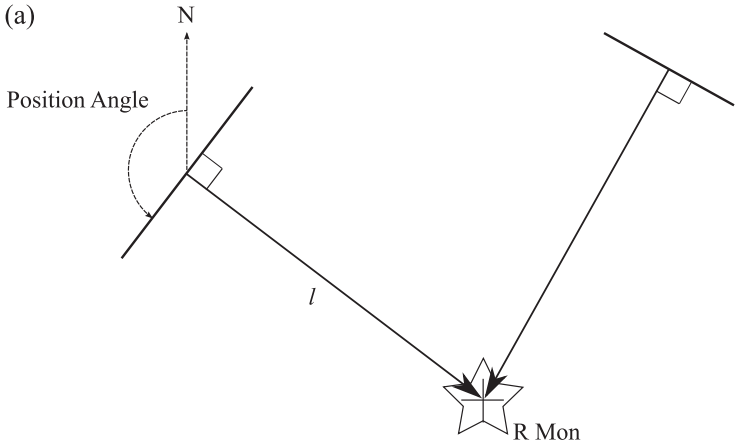

(b)

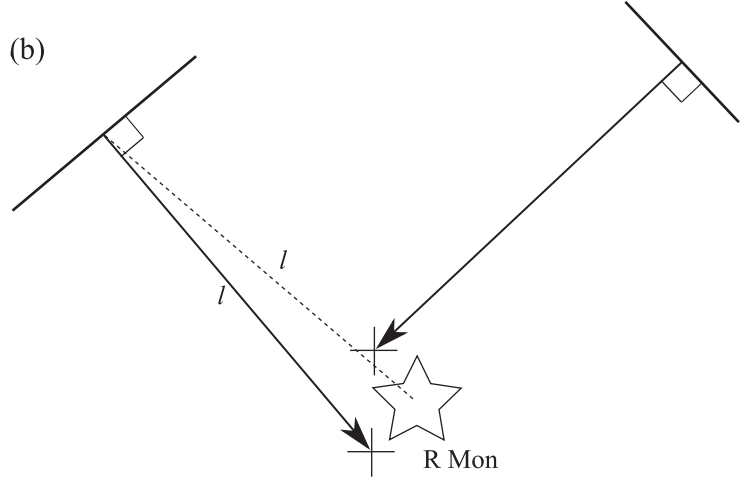

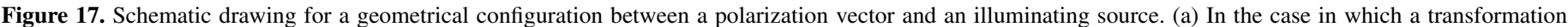

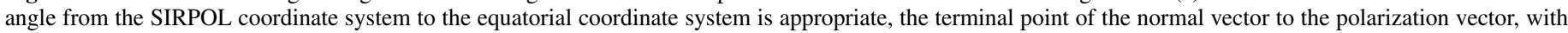

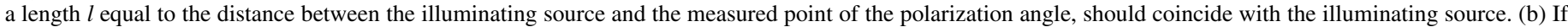
this is not the case, the terminal point does not coincide with the illuminating source.

do not exactly coincide with the illuminating source and are scattered around the illuminating source. If the transformation angle is not appropriate, scattering of the terminal points should become larger. Therefore, to determine the most appropriate transformation angle, we test the transformation angles with a range of $101^{\circ}-109^{\circ}$. Here we used only the polarization vectors with polarization degrees of $\gtrsim 3 \%$ (red vectors in Figure 16). As indicated in Figure 18, the transformation angles of $104^{\circ}-106^{\circ}$ appear to be more appropriate than those of $101^{\circ}-103^{\circ}$ and $107^{\circ}-109^{\circ}$. In $J$ - and $K_{\mathrm{s}}$-band analyses that were executed in the same way as the $H$-band analysis, the transformation angle of $104^{\circ}-106^{\circ}$ is also likely to be appropriate. We conclude that the transformation angle of $105^{\circ}$ reported by Kandori et al. (2006) is valid at present, and therefore we adopted $105^{\circ}$ as the transformation angle of SIRPOL in this paper. 


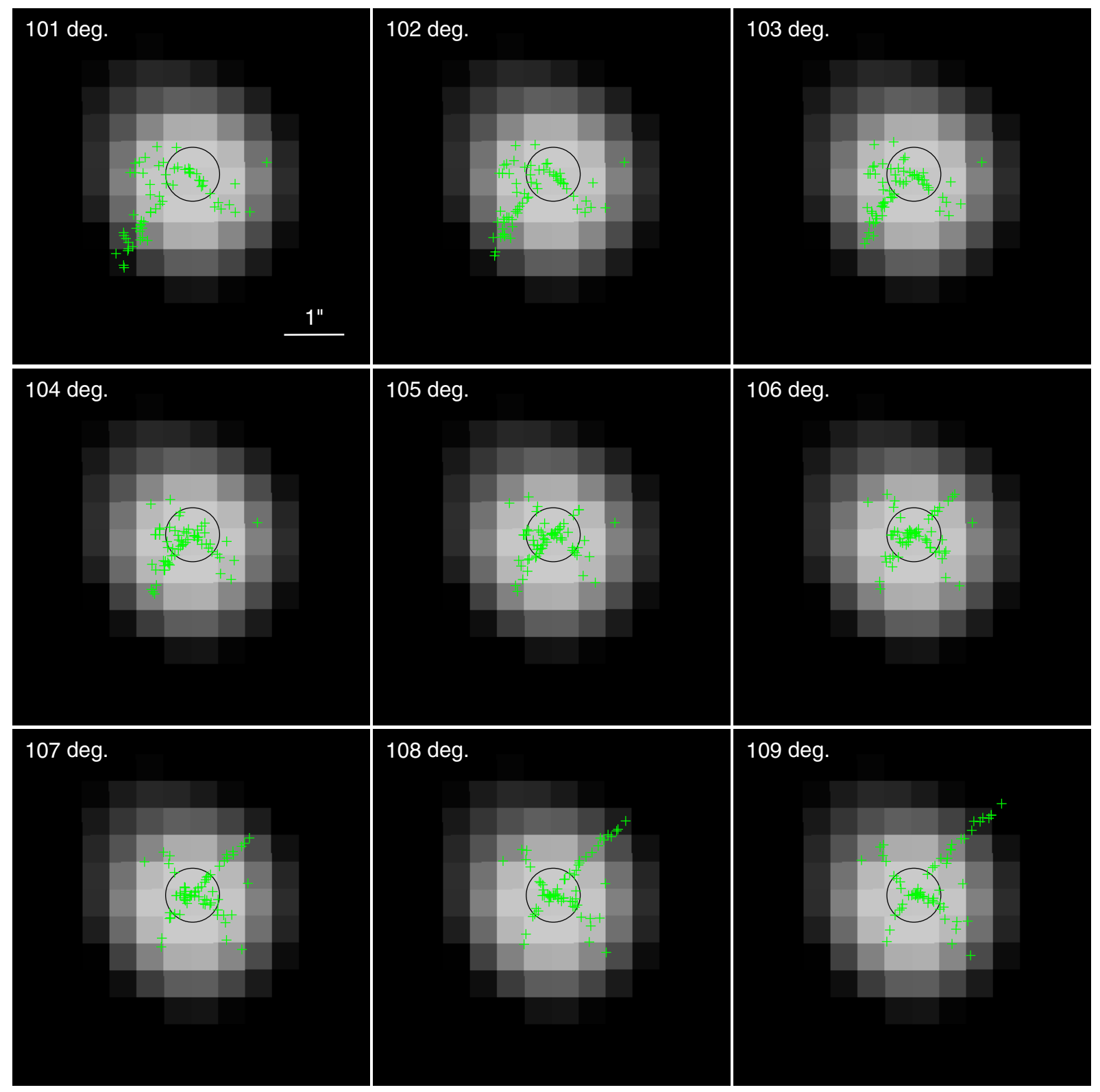

Figure 18. Distribution of the terminal points of the normal vectors to the polarization vectors (see the text). The center of the black circle shows the position of $\mathrm{R}$ Mon. In the lower right corner of the $101^{\circ}$ panel, the scale size of $1^{\prime \prime}$ is shown.

\section{REFERENCES}

Benjamin, R. A., Churchwell, E., Babler, B. L., et al. 2003, PASP, 115, 953 Bessell, M. S., \& Brett, J. M. 1988, PASP, 100, 1134

Carey, S. J., Norinaga-Crespo, A., Mizuno, D. R., et al. 2009, PASP, 121, 76 Chandrasekhar, S., \& Fermi, E. 1953, ApJ, 118, 113

Chini, R., \& Wargau, W. F. 1998, A\&A, 329, 161

Dickman, R. L. 1978, ApJS, 37, 407

Elmegreen, B. G. 1998, in ASP Conf. Ser. 148, Origins, ed. C. E. Woodward, J. M. Shull, \& H. A. Thronson, Jr. (San Francisco, CA: ASP), 150

Elmegreen, B. G. 2011, in Triggered Star Formation, ed. C. Charbonnel \& T. Montmerle (EAS Publ. Ser. 51; Cambridge: Cambridge Univ. Press), 45

Fitzpatrick, R. 2014, Plasma Physics: An Introduction (Boca Raton, FL: CRC Press), 169

Georgelin, Y. M., Boulesteix, J., Georgelin, Y. P., et al. 1987, A\&A, 174, 257

Kandori, R., Kusakabe, N., Tamura, M., et al. 2006, Proc. SPIE, 6269, 159

Kenyon, S. J., \& Hartmann, L. 1995, ApJ, 101, 117

Kessel-Deynet, O., \& Burkert, A. 2003, MNRAS, 338, 545

Kusakabe, N., Tamura, M., Kandori, R., et al. 2008, AJ, 136, 621

Lefloch, B., \& Lazareff, B. 1994, A\&A, 289, 559

Mackey, J., \& Lim, A. J. 2010, MNRAS, 403, 714

Mackey, J., \& Lim, A. J. 2011, MNRAS, 412, 2079
Meyer, M. R., Calvet, N., \& Hillenbrand, L. A. 1997, AJ, 114, 288

Miao, J., Sugitani, K., White, G. J., \& Nelson, R. P. 2010, ApJ, 717, 658

Miao, J., White, G. J., Nelson, R., et al. 2006, MNRAS, 369, 143

Miao, J., White, G. J., Thompson, M. A., \& Nelson, R. P. 2009, ApJ, 692,382

Minchin, N. R., Hough, J. H., McCall, A., et al. 1991, MNRAS, 249, 707

Motoyama, K., Umemoto, T., Shang, H., \& Hasegawa, T. 2013, ApJ, 766, 50

Nagashima, C., Nagayama, T., Nakajima, Y., et al. 1999, in Star Formation 1999, ed. T. Nakamoto (Nobeyama: Nobeyama Radio Observatory), 397

Nagayama, T., Nagashima, C., Nakajima, Y., et al. 2003, Proc. SPIE, 4841, 459

Nakano, T., \& Nakamura, T. 1978, PASJ, 30, 671

Oort, J. H., \& Spitzer, L. 1955, ApJ, 121, 60

Ostriker, E. C., Stone, J. M., \& Gammie, C. F. 2001, ApJ, 546, 980

Panagia, N. 1973, AJ, 78, 9

Parker, Q. A., Phillipps, S., Pierce, M. J., Hartley, M., et al. 2005, MNRAS, 362,689

Rathborne, J. M., Burton, M. G., Brooks, K. J., et al. 2002, MNRAS, 331, 85

Skrutskie, M. F., Cutri, R. M., Stiening, R., et al. 2006, AJ, 131, 1163

Stahler, S. W., \& Palla, F. 2004, in The Formation of Stars (Weinheim: Wiley)

Sugitani, K., Fukui, Y., \& Ogura, K. 1991, ApJ, 77, 59

Sugitani, K., \& Ogura, K. 1994, ApJS, 92, 163 
Sugitani, K., Watanabe, M., Tamura, M., et al. 2007, PASJ, 59, 507

Tamura, M., Kandori, R., Hashimoto, J., et al. 2007, PASJ, 59, 467

Testi, L., Palla, F., \& Natta, A. 1998, A\&A, 133, 81

Thompson, M. A., Urquhart, J. S., \& White, G. J. 2004, A\&A, 415, 627

Urquhart, J. S., Morgan, L. K., \& Thompson, M. A. 2009, A\&A, 497, 789

Urquhart, J. S., White, G. J., Pilbratt, G. L., \& Fridlund, C. V. M. 2003, A\&A, 403, 193
Wainscoat, R. J., Cohen, M., Volk, K., et al. 1992, ApJS, 83, 111

Wardle, J. F. C., \& Sramek, R. A. 1974, ApJ, 189, 399

White, G. J., Nelson, R. P., Holland, W. S., et al. 1999, A\&A, 342, 233

Williams, R. J. R., Ward-Thompson, D., \& Whitworth, A. P. 2001, MNRAS, 327, 788

Yamaguchi, N., Mizuno, N., Saito, H., et al. 1999, PASJ, 51, 775

Yamaguchi, R., Saito, H., Mizuno, N., et al. 1999, PASJ, 51, 791 